\title{
Signaling Pathways Controlling Induced Resistance to Insect Herbivores in Arabidopsis
}

\author{
Natacha Bodenhausen and Philippe Reymond \\ Department of Plant Molecular Biology, University of Lausanne, 1015 Lausanne, Switzerland \\ Submitted 22 January 2007. Accepted 22 June 2007.
}

\begin{abstract}
Insect attack triggers changes in transcript level in plants that are mediated predominantly by jasmonic acid (JA). The implication of ethylene (ET), salicylic acid (SA), and other signals in this response is less understood and was monitored with a microarray containing insect- and defense-regulated genes. Arabidopsis thaliana mutants coil-1, ein2-1, and sid2-1 impaired in JA, ET, and SA signaling pathways were challenged with the specialist small cabbage white (Pieris rapae) and the generalist Egyptian cotton worm (Spodoptera littoralis). JA was shown to be a major signal controlling the upregulation of defense genes in response to either insect but was found to suppress changes in transcript level only in response to $P$. rapae. Larval growth was affected by the JA-dependent defenses, but $S$. littoralis gained much more weight on coil-1 than $P$. rapae. ET and SA mutants had an altered transcript profile after $S$. littoralis herbivory but not after $P$. rapae herbivory. In contrast, both insects yielded similar transcript signatures in the abscisic acid (ABA)-biosynthetic mutants aba2-1 and aba3-1, and ABA controlled transcript levels both negatively and positively in insect-attacked plants. In accordance with the transcript signature, $S$. littoralis larvae performed better on aba2-1 mutants. This study reveals a new role for $A B A$ in defense against insects in Arabidopsis and identifies some components important for plant resistance to herbivory.
\end{abstract}

Plants are sessile organisms that cannot escape unfavorable conditions such as high insect density. Therefore, plants have evolved sophisticated strategies to cope with attack by herbivorous insects. These include both constitutive (e.g., trichomes, toxic secondary compounds, and thick walls) and inducible defenses. The latter can be subdivided into indirect (production of volatile odor blends to attract natural enemies of the attacker) (Dicke et al. 2003) and direct defenses (Karban and Baldwin 1997; Walling 2000). Direct defenses include the production of antidigestive proteins, toxic secondary compounds, proteases, or lectins that are active in the midgut and slow down or stop insect growth or development. Direct defenses have been studied initially in tomato, where caterpillar attack and mechanical wounding lead to the production of proteinase inhibitors that inhibit normal insect digestive activity (Ryan 1990). Numerous studies have further confirmed that chewing insects activate defense responses in most plant species investigated, including cultivated crops such as tomato

Corresponding author: Philippe Reymond; Telephone + 412169241 90; Fax +41 21692 4195; E-mail: philippe.reymond@unil.ch

* The $\boldsymbol{e}$-Xtra logo stands for "electronic extra" and indicates additional information is available online. Three supplemental tables are published online.
(Howe et al. 1996), potato (Johnson et al. 1989), maize (Pechan et al. 2002), or poplar (Constabel et al. 2000).

Unlike chewing insects, aphids and whiteflies have a stealthy feeding behavior that causes minimal tissue damage as they access the vascular tissue to feed on the phloem content (Thompson and Goggin 2006; Walling 2000). Work on plantaphid interactions has shown that defenses genes are activated that are known to act against bacterial and fungal pathogens (Fidantsef et al. 1999; Moran and Thompson 2001; Moran et al. 2002). Studies in squash and tomato revealed that defense gene activation in response to the silverleaf whitefly (Bemisia tabaci type B) involves known and novel defense signal transduction pathways (van de Ven et al. 2000; Walling 2000).

Herbivorous insects can be classified according to their feeding behavior and the amount of wounding that they produce: phloem feeders cause little wounding, chewing insects remove strips of leaves or make round holes, and cell-content feeders such as thrips feed on the content of cells but leave the damaged tissue on the plant. Another classification depends on the diet specialization. Some insects are generalists, feeding on members of several taxa, but most insects have narrow diets, feeding on plants from one or two taxonomic groups or even from one single species (Schoonhoven et al. 1998). According to the coevolutionary model, herbivorous insects have adapted to the host defenses and they are thought to overcome part of plant defense responses by detoxifying host gene products (Ehrlich and Raven 1964). In support of this model, two species of caterpillars feeding on members of the Brassicacae family, Plutella xylostella and Pieris rapae, were found to possess enzymes in the gut that help to detoxify glucosinolates (Ratzka et al. 2002; Wittstock et al. 2004). In contrast, P. rapae larvae are sensitive to high concentrations of isothiocyanates, a product of glucosinolate degradation (Agrawal and Kurashige 2003). Generalist insects, which might not possess any special detoxifying enzymes, are expected to be more sensitive to the same defenses. For example, the generalist beet armyworm (Spodoptera exigua) gained much more weight on transgenic potato plants lacking 13-lipoxygenase than the specialist potato Colorado beetle (Leptinotarsa decemlineata) (Royo et al. 1999). Moreover, resistance to the generalist Trichoplusia ni correlated with quantitative trait loci for glucosinolates, whereas no such correlation was observed for the specialist Plutella xylostella (Kliebenstein et al. 2002).

In recent years, development of global transcript profiling methods has allowed the identification of genes differentially expressed after insect attack (Korth 2003; Thompson and Goggin 2006). The interaction between the specialist caterpillar P. rapae and Arabidopsis thaliana has been used as a model system to study direct defenses at the molecular level and revealed substantial gene expression changes (De Vos et al. 2005; Reymond et al. 2000, 2004). In another model system, 
the molecular responses of Nicotiana attenuata to attack by the specialist herbivore Manduca sexta yielded transcript signatures illustrating the complex nature of the plant's response to different stress combinations (Hui et al. 2003; Schmidt et al. 2005; Voelckel and Baldwin 2004). Transcriptome analyses after aphid or whitefly feeding also identified substantial changes in gene expression and the transcript signature was found to be similar to a pathogen response (De Vos et al. 2005; Kempema et al. 2007; Moran et al. 2002; Thompson and Goggin 2006; Voelckel et al. 2004; Zhu-Salzman et al. 2004). When comparing expression profiling of Arabidopsis plants exposed to chewing insects, cell-content-feeding thrips, or phloem-feeding aphids, large expression changes specific to each attacker were identified, underscoring the complexity of the response to insect attack (De Vos et al. 2005). Similarly, silverleaf whitefly infestation triggered transcriptome changes very different from those induced by aphids and chewing insects (Kempema et al. 2007). On the other hand, the comparison of Arabidopsis responses to the specialist $P$. rapae and to the generalist $S$. littoralis revealed strikingly similar transcript profiles (Reymond et al. 2004).

Defense responses in plants are mediated by an interconnected network of signal transduction pathways depending mainly on the small regulators jasmonic acid (JA), salicylic acid (SA), and ethylene (ET) (Glazebrook 2001; Katagiri 2004; Reymond and Farmer 1998; Pieterse and van Loon 1999). JA, a fatty-acid-derived plant hormone, has been shown to play a major signaling role in defense against chewing and phloem-feeding insects in tomato (Cooper and Goggin 2005; Howe et al. 1996; Li et al. 2004), Medicago spp. (Gao et al. 2007), native tobacco (Kessler et al. 2004), and Arabidopsis (Ellis et al. 2002; McConn et al. 1997; Reymond et al. 2004; Zarate et al. 2007). Arabidopsis mutants impaired in either the synthesis or the perception of JA, SA, or ET have been crucial in addressing the respective role of these molecules during insect attack. For instance, it was shown using the coronatine insensitive 1 (coil-1) mutant that a large proportion of the genes induced by $P$. rapae require a functional jasmonate pathway and that larval performance of this specialist insect was enhanced on coil-1 plants (Reymond et al. 2004). A mutant impaired in the conversion of the JA precursor 12-oxo-phytodieonic acid (OPDA) to JA exhibited wild-type resistance to the dipteran Bradysia impatiens, illustrating differential roles for jasmonate family members during herbivory (Stintzi et al. 2001). In the case of phloem-feeders, coil-1 allowed an increased aphid population growth (Ellis et al. 2002) and an accelerated silverleaf whitefly nymph development (Zarate et al. 2007). Thus, the JA pathway is emerging as a major player in the activation of genes involved in induced defenses (De Vos et al. 2005; Reymond et al. 2004; Schenk et al. 2000) and in the release of volatiles responsible for indirect defenses (Thaler et al. 2002; Van Poecke and Dicke 2002).

The implication of ET and salicylate pathways in defense against insects also is documented. The generalist Egyptian cotton worm ( $S$. littoralis) was shown to cause more damage on wild-type Arabidopsis plants than on ethylene insensitive 2 (ein2-1) mutant plants, whereas no difference was observed with the specialist diamondback moth (Plutella xylostella) (Stotz et al. 2000). ET released during the interaction between the specialist herbivore $M$. sexta and its native host $N$. attenuata reduced the accumulation of nicotine, a direct defense, without affecting the release of volatile terpenoids, an indirect defense (Kahl et al. 2000). A mutant in the NON-EXPRESSER OF PATHOGENESIS-RELATED GENES1 (NPRI, also known as NIM1) locus, impaired in the SA-induced defense gene expression (Cao et al. 1994), showed enhanced resistance to $S$. littoralis, suggesting that, in wild-type plants, S. littoralis larvae activate the SA pathway to render plants more susceptible (Stotz et al. 2002). Furthermore, the SA-compromised niml-1 mutant showed reduced feeding by the beet armyworm, S. exigua (Cipollini et al. 2004). In another study, larvae of the generalist cabbage looper (T. ni) gained less weight on nprl-1 and $S A$ induction deficient2 (sid2-1) mutants (Cui et al. 2002). Silverleaf whitefly nymph development was shown to be accelerated on SA overepression mutants whereas it was delayed on nprl-1 and SA-degrading NahG plants (Zarate et al. 2007). However, the implication of SA and ET pathways in defense against aphids is less clear (Thompson and Goggin 2006). Thus, although both ET and SA pathways seem to play a role in insect defense, very few studies assessed the role of these pathways in the control of insect-regulated genes at a global scale.

The plant hormone abscisic acid (ABA) plays a major role in the control of seed germination and in the response to abiotic stress caused by drought, salt, and wounding (Christmann et al. 2006). ABA recently has been implicated in the regulation of defense-related genes (Anderson et al. 2004; Lorenzo et al. 2004), and there is accumulating evidence that ABA levels correlate with disease resistance in several plant-pathogen interactions (Mauch-Mani and Mauch 2005). For example, ABA controls priming against an oomycete (Ton et al. 2005). Because of its implication during wounding, ABA is thought to modulate response to insect feeding (de Bruxelles and Roberts 2001; Walling 2000). Tomato mutant plants with reduced ABA levels were found to be more susceptible to $S$. exigua feeding (Thaler and Bostock 2004). However, the role of ABA in resistance to insects has not been studied in Arabidopsis.

The aim of this study was to investigate the role of known signaling pathways involved in biotic and abiotic stresses in the response to two herbivores with different diet breadth. Arabidopsis mutants compromised in JA, SA, ET, and ABA pathways were challenged with $P$. rapae and $S$. littoralis larvae and their transcript profiles monitored with a dedicated DNA microarray containing insect- and defense-regulated genes. Changes in transcript levels in response to the specialist and generalist insects were not identical in the JA, SA, and ET mutants, whereas they were quite similar in the ABA mutant. Insect performance also was evaluated on the same mutants, with the goal to assess the contribution of different signaling pathways on plant resistance to herbivores.

\section{RESULTS}

\section{Expression changes in response to $P$. rapae and $S$. littoralis in Arabidopsis signaling mutants.}

To evaluate the role of known defense signaling pathways in gene expression changes after herbivory in Arabidopsis, a dedicated DNA microarray containing genes regulated by $P$. rapae or $S$. littoralis was constructed. These genes were identified in a study using a $12 \mathrm{~K}$ Arabidopsis cDNA microarray (Reymond et al. 2004). This $12 \mathrm{~K}$ microarray contained a random set of 12,135 expressed sequence tags (EST) from the Arabidopsis Biological Resource Center. Data from several experiments were used to select insect-regulated genes: i) wild-type plants after $5 \mathrm{~h}$ of herbivory by $P$. rapae and $S$. littoralis, ii) jasmonateinsensitive mutant coil-1 gll after 5 h of herbivory by $P$. rapae, and iii) wild-type plants $24 \mathrm{~h}$ after the end of $5 \mathrm{~h}$ of herbivory by $P$. rapae (Reymond et al. 2004). In addition, known pathogen- and stress-responsive genes were included as well as housekeeping genes used for normalization (Supplemental Table 1). In all, 279 cDNAs, representing 222 unique genes, were spotted on the microarray. Thus, although this microarray does not contain all genes that are regulated by insect herbivory in Arabidopsis, it provides a useful set of marker genes to assess the implication of signaling pathways in insect defense. 
For each experiment, the relative abundance of RNAs was measured and expression ratios were calculated from untreated and insect-attacked plants using several independent biological replicates. Upregulated or downregulated genes were defined as genes with an expression ratio $\geq 2$ or $\leq 2$, and the statistical significance of the induction was calculated by comparing expression ratios from insect-attacked/control experiments with expression ratios from control/control experiments (Student's $t$ test, $P<0.05)$. To identify genes differentially regulated by herbivory between wild-type and signaling mutants, a statistical test was performed comparing expression ratios calculated from wild-type plants with expression ratios from mutant plants (Student's $t$ test, $P<0.05$ ) (discussed below). To calculate a false discovery rate (FDR) for each experiment, a $q$ value was computed (Storey and Tibshirani 2003). The FDRs at $P=0.05$ ranged from 0.1 to $2.5 \%$ (discussed below), indi- cating that the lists of genes considered as significantly regulated are robust and giving confidence to the interpretation of the data.

First, gene expression profiles were compared in wild-type plants and in the null mutant coil-1 in response to herbivory by either $P$. rapae or $S$. littoralis. Feeding by $P$. rapae increased the RNA levels of 87 genes in Col-0, of which 40 showed no change in RNA abundance in coil-1 plants (Fig. 1A; Supplemental Table 2). More than $90 \%$ of the genes upregulated also were upregulated in our previous study, from which the cDNAs were selected (Reymond et al. 2004), and the same genes were dependent on coil-1, illustrating the robustness of our microarray analyses (data not shown). Feeding by $S$. littoralis upregulated 119 genes in Col-0, of which 71 showed no change in RNA abundance in coil-1 (Fig. 1B), indicating that $\mathrm{JA}$ is also a major signal in the response to herbivory by a gen-
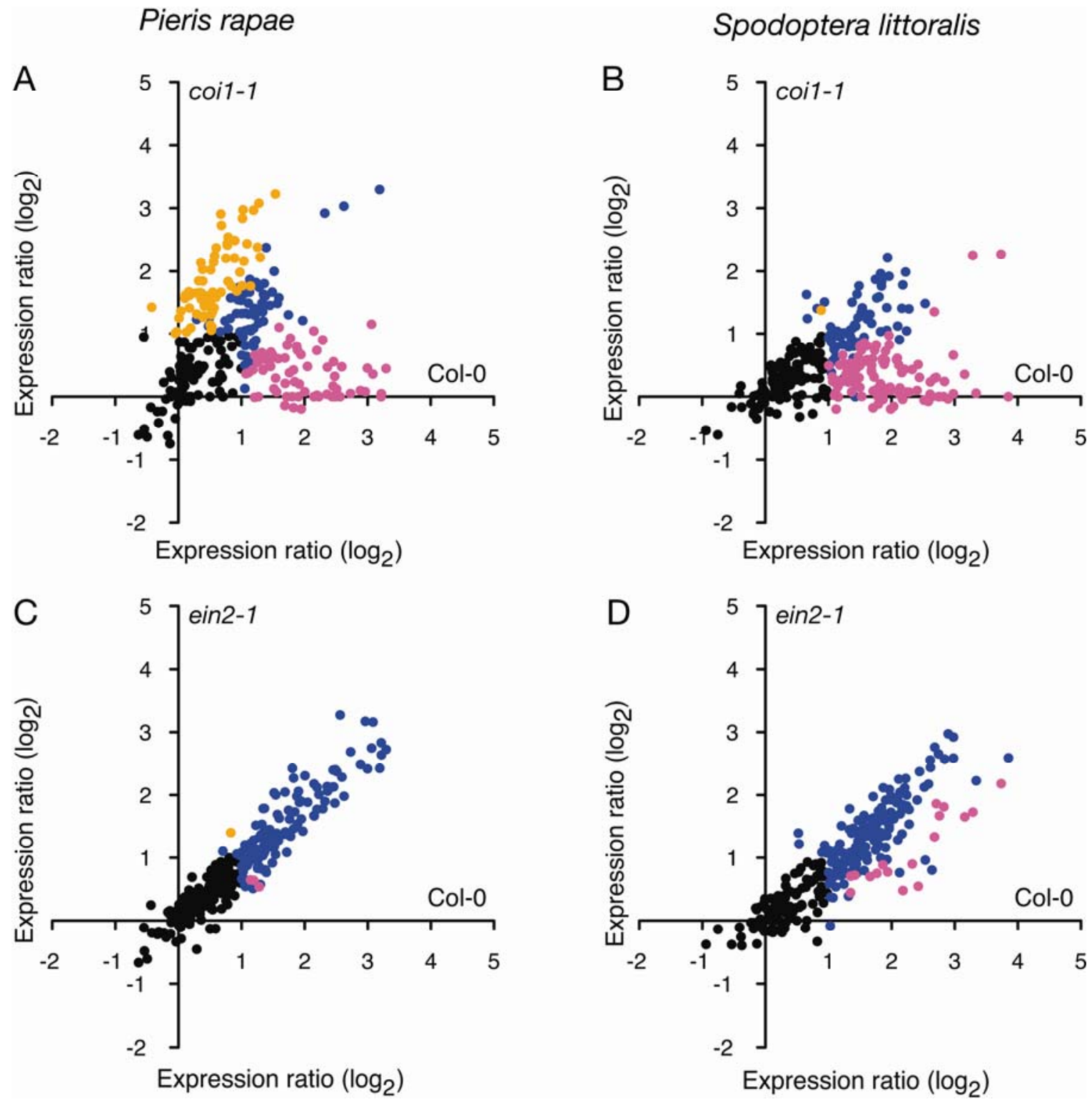

Fig. 1. Changes in transcript level in wild-type and mutant Arabidopsis plants after challenge for $5 \mathrm{~h}$ with $\mathbf{A}$ and $\mathbf{C}$, Pieris rapae and $\mathbf{B}$ and D, Spodoptera littoralis. Relative changes in RNA abundance after challenge with insects were measured in Col-0 and in different signaling mutant plants: A and B, coil-1 and $\mathbf{C}$ and $\mathbf{D}$, ein2-1. Mean expression ratios calculated from experiments comparing challenged and unchallenged Col-0 plants (eight independent replicates for $P$. rapae and six independent replicates for $S$. littoralis) were plotted against mean expression ratios between challenged and unchallenged mutant plants (four independent replicates). Black dots represent genes that showed no changes in transcript abundance, blue dots represent genes that showed a higher transcript level in both genotypes in response to herbivory but with no statistical difference between genotypes, magenta dots represent genes that showed a significantly larger change in transcript level in wild-type than in mutant plants, and orange dots represent genes that showed a significantly larger change in transcript level in mutant than in wild-type plants (Student's $t$ test). 
eralist insect. Moreover, there was a large overlap between the two sets of COI1-dependent genes (Table 1). The genes depending on a functional COI1 protein encoded mostly defense proteins and oxylipin synthesis enzymes (Table 1).

$P$. rapae increased the RNA level of 45 genes significantly in coil-1 plants but not in Col-0 plants (Fig. 1A; Table 1), suggesting that, upon herbivory, JA blocks the upregulation of these transcripts. Strikingly, this suppression was not found with S. littoralis feeding (Fig. 1B). Among those genes, two functional categories were clearly identified: defense against pathogens (NPR1, PR5, MPK3, syntaxin SYP121, harpininduced protein 1 , class IV chitinase, and toll interleukin 1 receptor-nucleotide binding site [TIR-NBS] class disease resistance protein) and calcium signaling (calmodulin CAM3, calcium-binding EF-hand protein, and calmodulin-related TCH3)
(Table 1). Other genes included transcription factors, dehydration-induced genes, and genes of various functions. The majority of these JA-suppressed genes were upregulated only in the coil-1 mutant during $P$. rapae herbivory, whereas six genes also were upregulated during $S$. littoralis herbivory in both wild-type and coil-1 plants (Table 1).

The ET pathway also is known to be involved in plant defense responses (Kunkel and Brooks 2002) and some genes require the cooperation of both JA and ET for their induction (Reymond and Farmer 1998). To assess the role of ET in the control of insect-responsive genes, the ET-insensitive null mutant ein2-1 (Guzman and Ecker 1990) was challenged with P. rapae larvae for 4 to $5 \mathrm{~h}$ and the transcript pattern was compared with that of Col-0 plants. Surprisingly, almost no difference was found between the two genotypes (Fig. 1C). One gene encoding a
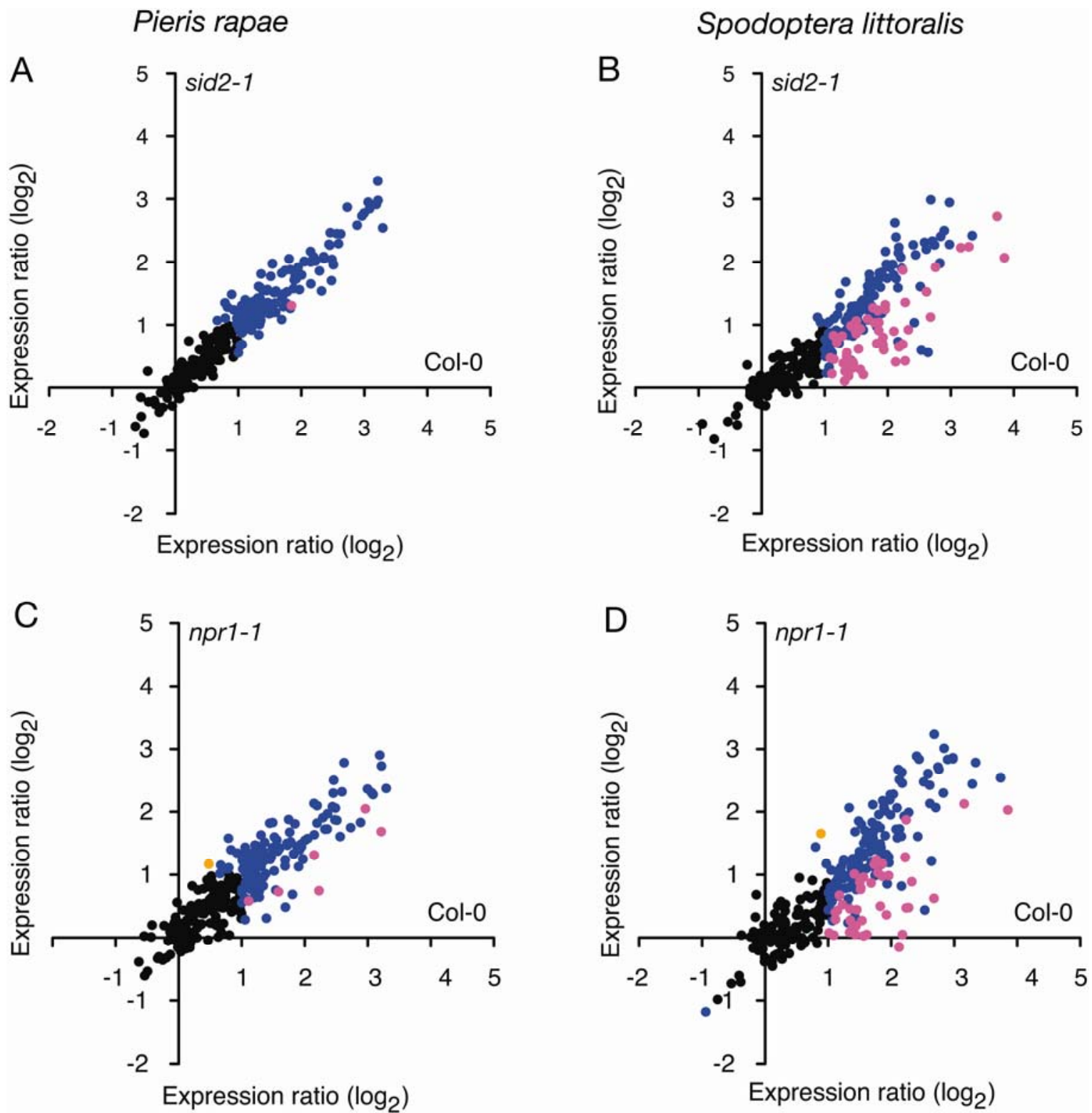

Fig. 2. Changes in transcript level in wild-type and mutant Arabidopsis plants after challenge for 5 h with A and C, Pieris rapae and B and D, Spodoptera littoralis. Relative changes in RNA abundance after challenge with insects were measured in Col-0 and in different signaling mutant plants: A and B, sid2-1 and $\mathbf{C}$ and D, nprl-1. Mean expression ratios calculated from experiments comparing challenged and unchallenged Col-0 plants (eight independent replicates for $P$. rapae and six independent replicates for $S$. littoralis) are plotted against mean expression ratios between challenged and unchallenged mutant plants (four independent replicates). Black dots represent genes that showed no changes in transcript abundance, blue dots represent genes that showed a higher transcript level in both genotypes in response to herbivory but with no statistical difference between genotypes, magenta dots represent genes that showed a significantly larger change in transcript level in wild-type than in mutant plants, and orange dots represent genes that showed a significantly larger change in transcript level in mutant than in wild-type plants (Student's $t$ test). 
late embryogenesis abundant protein (LEA14, At1g01470) showed a significantly higher transcript level in ein2-1 than in Col-0, and two lectins (At3g15356 and At3g16530) showed a significantly higher transcript level in Col-0 than in ein2-1 plants (Table 1 ). In the case of $S$. littoralis, RNAs of only $10 \%$ of the insect-regulated genes were significantly more abundant in Col-0 than in ein2-1 (Fig. 1D). Examples of these genes encode a hydroperoxide lyase (HPL1, At4g15440), a hydroxyjamonates sulfotransferase (At5g07010), an NAC transcription factor (At1g52890), and a ribonuclease (RNS1, At2g02990) (Table 1).

Given the importance of SA in plant defense responses (Shah 2003), the expression of insect-regulated genes was analyzed in the SA-deficient mutant sid2-1. This mutant has normal SA levels in resting leaves but fails to accumulate SA upon challenge with pathogens (Nawrath and Metraux 1999). Plants challenged with $P$. rapae larvae for 4 to $5 \mathrm{~h}$ showed a transcript pattern almost identical in sid2-1 and in Col-0 plants (Fig. 2A). Only one gene encoding a tyrosine aminotransferase (At2g24850) showed a significantly higher transcript level in Col-0 than in sid2-1 plants. The gene expression changes also were studied in the SA-perception mutant nprl-1. Mutants with defects in NPR1 fail to respond to various SAR-inducing treatments, displaying low transcript abundance of $P A T H O$ GENESIS-RELATED (PR) genes and exhibiting increased susceptibility to biotrophic infections (Cao et al. 1994). Again, there was very little difference between mutant and wild-type plants in response to $P$. rapae (Fig. 2C). In contrast, when sid2-1 was challenged with $S$. littoralis larvae, RNAs of a third of insect-regulated genes were statistically more abundant in Col-0 than in sid2-1 plants (Fig. 2D). Similar results were obtained with the nprl-1 mutant (Fig. 2B). Some genes were not

Table 1. Representative jasmonic acid (JA)- and ethylene (ET)-regulated genes in response to Pieris rapae and Spodoptera littoralis feeding ${ }^{\mathrm{a}}$

\begin{tabular}{|c|c|c|c|c|c|c|c|}
\hline \multirow[b]{2}{*}{ AGI } & \multirow[b]{2}{*}{ Description } & \multicolumn{3}{|c|}{ Pieris rapae } & \multicolumn{3}{|c|}{ Spodoptera littoralis } \\
\hline & & Col-0 & coil-1 & ein2-1 & Col-0 & coil-1 & $\operatorname{ein} 2-1$ \\
\hline \multicolumn{8}{|c|}{ JA- and ET-regulated } \\
\hline At1g52890 & No apical meristem (NAM) protein & $2.27 * * *$ & $1.34 * * *$ & $1.42 * * *$ & $5.02 * * *$ & $1.35^{*}$ & $1.86^{*}$ \\
\hline At2g02990 & Ribonuclease, RNS1 & $3.49 * *$ & 1.00 & $5.38 * * *$ & $5.36 * *$ & $1.43^{*}$ & 1.46 \\
\hline At2g29450 & Glutathione $S$-transferase (GSTU5) & $5.69 * * *$ & 1.09 & $5.19 * * *$ & $6.53 * * *$ & 1.23 & $3.63 * *$ \\
\hline At4g15440 & Hydroperoxide lyase (HPL1) & $3.74 * * *$ & 1.00 & $4.17 * *$ & $3.63 * * *$ & 1.00 & $1.85^{*}$ \\
\hline At5g03210 & Expressed protein & $2.57 * * *$ & $3.49 *$ & 3.45 & $6.40 * * *$ & $2.55^{*}$ & $2.51 * *$ \\
\hline At5g07010 & Hydroxyjamonate sulfotransferase & $5.60 * * *$ & 1.00 & $5.30 * * *$ & $7.09 * * *$ & 1.00 & $3.51 * *$ \\
\hline At5g64310 & Arabinogalactan-protein (AGP1) & $2.19 * * *$ & $3.65 * *$ & 1.46 & $3.83 * * *$ & $4.65^{*}$ & $1.71 *$ \\
\hline \multicolumn{8}{|c|}{ COI1-suppressed } \\
\hline At1g08930 & Sugar transporter & 1.31 & $2.40 * * *$ & 1.24 & 1.25 & 1.30 & 1.59 \\
\hline At1g20440 & Dehydrin (COR47) & 1.37 & $3.04 * *$ & $1.61 *$ & $2.06 * * *$ & $1.83^{*}$ & 1.57 \\
\hline At1g20450 & Dehydrin (ERD10) & $1.73^{*}$ & $5.81 * * *$ & 1.79 & $3.00 * *$ & $2.49 *$ & 2.25 \\
\hline At1g27730 & Salt-tolerance zinc finger protein & $1.71 *$ & $5.33 * * *$ & 1.20 & $4.84 * *$ & $2.63 * *$ & 3.41 \\
\hline At1g28330 & Dormancy-associated protein -related & 1.12 & $3.18 * * *$ & $1.31 *$ & $1.38 *$ & $1.61 * *$ & 1.68 \\
\hline At1g42990 & bZIP family transcription factor & 1.07 & $2.96 * * *$ & 1.21 & $1.77 * *$ & $1.61 *$ & 1.10 \\
\hline At1g51700 & Dof zinc finger protein & 1.13 & $2.66 * *$ & 1.09 & $1.49 * *$ & $1.71 * *$ & $1.19 *$ \\
\hline At1g64280 & Regulatory protein (NPR1) & $1.22 * *$ & $2.49 * *$ & $1.33 * *$ & $1.25 * *$ & $1.44 *$ & $1.09^{*}$ \\
\hline Atlg72910/50 & $\begin{array}{l}\text { Disease resistance protein (toll interleukin } 1 \\
\text { receptor-nucleotide binding site class) }\end{array}$ & 1.29 & $3.14 * * *$ & 1.15 & 1.28 & 1.35 & 1.10 \\
\hline At1g75040 & Pathogenesis-related protein $5(\mathrm{PR}-5)$ & 1.52 & $5.16^{*}$ & 1.50 & 1.45 & 1.42 & 2.32 \\
\hline At2g22500 & Mitochondrial carrier protein family & $1.51^{*}$ & $2.99 * *$ & 1.64 & $3.06 * * *$ & $2.35 * *$ & 1.94 \\
\hline At2g23810 & Senescence-associated protein family & 1.43 & $4.06 * *$ & 1.24 & $2.50 * *$ & 1.73 & 1.69 \\
\hline At $2 \mathrm{~g} 38530$ & Lipid transfer protein 2 (LTP 2) & $1.09 * * *$ & $2.04 * *$ & 1.12 & $1.57 * *$ & $3.09 *$ & 1.57 \\
\hline At2g41100 & Calmodulin-related protein $3(\mathrm{TCH} 3)$ & 1.61 & $6.62 * *$ & 1.24 & $2.90 * *$ & $2.66 * *$ & 2.95 \\
\hline At2g41430 & ERD15 protein & 1.46 & $3.17 * * *$ & 1.38 & $2.02 * *$ & 1.41 & 1.50 \\
\hline At2g47730 & Glutathione transferase (GST6/GSTF8) & $1.47 *$ & $4.43 * * *$ & 1.26 & $1.92 * * *$ & $2.84 * *$ & 2.29 \\
\hline At3g11820 & Syntaxin of plants SYP121 & $1.29 * *$ & $2.93 * *$ & 1.32 & $1.50 * *$ & $1.63 *$ & 1.43 \\
\hline At3g45640 & Mitogen-activated protein kinase (MPK3) & 1.13 & $3.06 * *$ & 1.16 & $1.45^{*}$ & $1.65^{*}$ & 1.16 \\
\hline At3g54420 & Glycosyl hydrolase (class IV chitinase) & 0.75 & $2.68 * *$ & 0.88 & 0.99 & 1.06 & 1.27 \\
\hline At3g55980 & Zinc finger transcription factor (PEI1) & 1.31 & $4.08 * *$ & 1.35 & $2.96^{*}$ & $1.92 *$ & 1.74 \\
\hline At3g56800 & Calmodulin & $1.48 * * *$ & $2.66 * *$ & $1.43^{*}$ & $1.56 * * *$ & $1.50 * *$ & 1.45 \\
\hline At4g17500 & ET responsive element binding factor 1 & 1.32 & $2.42 * * *$ & 1.22 & $1.48 *$ & 1.26 & $1.78 *$ \\
\hline At4g02380 & LEA protein family & $1.59 *$ & $7.50 * * *$ & 1.91 & $4.53 * *$ & $3.44 * *$ & 1.39 \\
\hline At4g32060 & Calcium-binding EF-hand family protein & 1.08 & $3.07 * * *$ & 0.99 & 1.25 & 1.11 & 1.47 \\
\hline At5g06320 & Harpin-induced protein 1 family & 1.25 & $3.60 * * *$ & 1.43 & $2.60 * * *$ & $2.12 *$ & 1.88 \\
\hline At5g22380 & No apical meristem (NAM) protein & 1.01 & $2.38 * *$ & 1.16 & 1.18 & 1.46 & 0.91 \\
\hline At5g26340 & Hexose transporter, putative & $1.71 *$ & $5.42 * *$ & $1.74 *$ & $2.20 * * *$ & $2.06^{*}$ & 1.79 \\
\hline At5g58430 & Leucine zipper-containing protein & 1.28 & $4.40 * *$ & 1.01 & 1.89 & 1.46 & 1.22 \\
\hline At5g66400 & Dehydrin RAB18-related protein & $1.15^{*}$ & $2.13 * * *$ & 1.16 & 1.19 & 1.37 & 1.22 \\
\hline \multicolumn{8}{|l|}{ JA-dependent } \\
\hline At1g05010 & ACC oxidase & $1.97 * * *$ & $1.82 *$ & $1.99 *$ & $2.01 * *$ & 1.41 & $2.16^{*}$ \\
\hline At1g12240 & $\beta$-Fructosidase & $2.56 * * *$ & $1.51 * *$ & $2.22 *$ & $3.58 * * *$ & $1.13^{*}$ & $3.28 * * *$ \\
\hline At1g15950 & Cinnamoyl-CoA reductase & $1.97 * * *$ & $2.04 * *$ & $2.05 *$ & $2.26 * * *$ & $1.42 *$ & $2.32 * *$ \\
\hline At1g17420 & Lipoxygenase (LOX3) & $7.79 * * *$ & 1.06 & $9.01 * *$ & $14.43 * * *$ & 1.00 & $6.03 * *$ \\
\hline At1g17620 & Expressed protein & $1.58 * * *$ & $1.42 * *$ & $1.43^{*}$ & $2.45^{* * *}$ & $1.36^{* *}$ & $1.92 * *$ \\
\hline At1g19570 & Dehydroascorbate reductase & $5.12 * * *$ & 1.03 & $4.40 * *$ & $4.52 * * *$ & 0.9 & $3.60 * * *$ \\
\hline At1g32640 & bHLH protein (RAP-1) (MYC2) & $3.00 * * *$ & $1.52 *$ & $2.41 *$ & $3.34 * * *$ & 1.07 & $2.47 * *$ \\
\hline
\end{tabular}

\footnotetext{
${ }^{a}$ Expression ratios are color coded according to intensity: yellow (from 2 to 4 ), light orange (from 4 to 6 ), dark orange (from 6 to 10 ), red ( $>10$ ). $* \mathrm{P}$ value $<0.05$, ** $\mathrm{P}$ value $<0.01, * * * \mathrm{P}$ value $<0.001$ ( $\mathrm{P}$ values were calculated on log-transformed ratios from treatment and control experiments, Student's $t$-test). Experiments with $P$. rapae in Col-0 and coil-1 were also performed in our previous study using a 12-K cDNA microarray (Reymond et al. 2004).
} 
upregulated in the sid2-1 or nprl-1 mutants in response to $S$. littoralis herbivory (expression ratio $<2$ ), which suggests that SA is necessary for their activation; including, for example, a zinc finger transcription factor (At3g55980), two calmodulinrelated genes (TCH2, At5g37770; and TCH3, At2g41100), and a lipid-transfer protein (At3g22600) (Table 2). Some genes showed a significantly higher transcript level in Col-0 than in sid2-1 and npr 1-1 mutants, indicating a partial control by SA; two examples of this class are LIPOXGENASE3 (LOX3, At1g17420) and an NAC transcription factor (At1g52890) (Table 2). Interestingly, most genes whose transcript abundance was controlled by SA after $S$. littoralis feeding also were COI1 dependent, including genes that were suppressed by $P$. rapae.

The differential RNA abundance of some genes in response to $P$. rapae and $S$. littoralis herbivory between Col-0 and coil1 or sid2-1 mutants was verified by quantitative real-time polymerase chain reaction (PCR) for three representative genes.
The syntaxin SYP121 gene (At3g11820) and a gene encoding a disease resistance protein of the TIR-NBS class (At1g72950) were induced only in response to $P$. rapae in coil-1 plants (Fig. 3). This confirmed the microarray data showing that JA signaling pathway suppressed the upregulation of some genes in response to $P$. rapae but not in response to $S$. littoralis. The TCH3 gene (At2g41100) showed a significantly higher RNA level in response to $S$. littoralis in Col-0 but not in sid2-1, confirming the control of its expression by the SA pathway, whereas the relative RNA abundance of this gene was not changed in response to $P$. rapae (Fig. 3).

Although ABA is considered to control mainly responses to abiotic stress, its implication in resistance to pathogens has been recently documented (Mauch-Mani and Mauch 2005), but its role in the response to insect herbivory in Arabidopsis is not characterized. Two mutants impaired in the last steps of ABA biosynthesis were tested; namely, aba2-1, whose gene

Table 1. Continued from preceding page

\begin{tabular}{|c|c|c|c|c|c|c|c|}
\hline \multirow[b]{2}{*}{ AGI } & \multirow[b]{2}{*}{ Description } & \multicolumn{3}{|c|}{ Pieris rapae } & \multicolumn{3}{|c|}{ Spodoptera littoralis } \\
\hline & & Col-0 & coi1-1 & ein2-1 & Col-0 & coil-1 & $\operatorname{ein} 2-1$ \\
\hline \multicolumn{8}{|c|}{ JA-dependent (Continued) } \\
\hline At1g45145 & Thioredoxin H-type 5 (TRX-H-5) & $1.24 * *$ & $1.02 *$ & $1.14^{*}$ & $2.16^{* * *}$ & $1.04 *$ & $1.45^{*}$ \\
\hline At1g51760 & Aminohydrolase & $6.02 * * *$ & $1.39 *$ & $4.88 *$ & $7.16 * * *$ & $1.18 *$ & $5.95 * * *$ \\
\hline At1g52400 & $\beta$-Glucosidase (BG1) & $5.14 * * *$ & $1.38 *$ & $4.18 * * *$ & $4.33 * * *$ & 1.42 & $4.77 * *$ \\
\hline At1g54010 & Myrosinase-associated protein & $3.29 *$ & 1.00 & $2.12 * *$ & $5.72 * *$ & 0.96 & $4.37 * * *$ \\
\hline At1g62660 & $\beta$-Fructosidase & $4.44 * * *$ & $1.00 * *$ & $3.17 * *$ & $3.27 * * *$ & $1.06 * *$ & $3.10 * * *$ \\
\hline At1g72450 & Expressed protein & $2.39 * * *$ & $1.46^{*}$ & $1.80 * *$ & $3.46^{* * * *}$ & 0.92 & $3.35^{* *}$ \\
\hline At1g72520 & Lipoxygenase (LOX4) & $2.91 * *$ & 1.23 & 4.01 & $6.23 *$ & 1.00 & $1.75^{*}$ \\
\hline At1g74100 & Desulfoglucosinolate sulfotransferase & $2.29 * * *$ & $1.74 * * *$ & $1.96^{*}$ & $3.21 * * *$ & $1.32 *$ & $2.68 * *$ \\
\hline At1g74950 & Expressed protein & $4.68 * * *$ & 1.00 & 4.02 & $4.39 * * *$ & 1.10 & $3.24 * * *$ \\
\hline At1g75220 & Integral membrane protein & $2.09 * *$ & $1.75 * * *$ & 2.12 & $2.42 * * *$ & $1.27 *$ & $2.07 *$ \\
\hline At1g76790 & $O$-methyltransferase & $6.63 * * *$ & 1.04 & $6.44 * *$ & $6.11 * * *$ & 1.03 & $5.88 * * *$ \\
\hline At2g06050 & 12-oxophytodienoate reductase (OPR3) & $9.79 * * *$ & 1.36 & 6.62 & $7.89 * * *$ & 1.58 & $7.58 * *$ \\
\hline At2g17840 & Senescence-associated protein 12 -related & $2.21 * * *$ & $1.44 *$ & $2.34 * *$ & $1.97 * *$ & 1.00 & $1.35^{*}$ \\
\hline At2g24850 & Aminotransferase & $3.59 * * *$ & 1.18 & 3.32 & $6.13 * * *$ & $1.18 *$ & $5.43 * * *$ \\
\hline At2g30870 & Glutathione $S$-transferase (ERD13/GSTF8) & $1.91 * * *$ & $1.93 * *$ & 1.67 & $2.22 * * *$ & 1.12 & $2.2 * *$ \\
\hline At2g36830 & Major intrinsic protein (MIP) family & $2.94 * * *$ & 1.05 & $2.62 * *$ & $2.63 * *$ & 1.75 & $2.74 *$ \\
\hline At $2 \mathrm{~g} 37180$ & Aquaporin PIP2.3 (RD28) & $3.54 * * *$ & 0.89 & 2.75 & $3.62 * * *$ & $1.51 *$ & $3.30 *$ \\
\hline At2g46370 & Auxin-responsive protein & $2.31 * *$ & 0.99 & $2.13 *$ & $2.58 * *$ & 1.05 & $1.72 *$ \\
\hline At2g47180 & Galactinol synthase & $1.73 * *$ & $1.43 *$ & 1.85 & $3.30 * * *$ & $1.24 *$ & $2.41 * *$ \\
\hline At3g11170 & Omega-3 fatty acid desaturase (FAD7) & $2.77 * * *$ & $1.63 * * *$ & 2.48 & $2.69 * * *$ & 1.15 & $2.63 * *$ \\
\hline At3g13110 & Serine acetyltransferase (SAT1) & $2.08 * *$ & 1.09 & $1.48^{*}$ & $2.14 * * *$ & 1.01 & $1.67 * *$ \\
\hline At3g16400 & Myrosinase-binding protein & $9.30 * * *$ & 1.03 & $6.23 * *$ & $6.42 * * *$ & 1.23 & $6.78 * * *$ \\
\hline At3g16410 & Myrosinase-binding protein & $5.47 * * *$ & $1.12 * * *$ & $5.28 * * *$ & $5.99 * * *$ & $0.95 * * *$ & $4.52 * * *$ \\
\hline At3g44320 & Nitrilase 3 & $2.16^{* * * *}$ & 1.30 & $1.90 * * *$ & $1.96 * * *$ & 1.07 & $2.11 *$ \\
\hline At3g44720 & Prephenate dehydratase & $2.73 * * *$ & $2.74 * *$ & 2.42 & $3.89 * * *$ & $1.96^{*}$ & $2.76 * *$ \\
\hline At3g45140 & Lipoxygenase (LOX2) & $3.22 * *$ & 0.91 & 2.59 & $3.54 * *$ & 1.15 & $2.64^{*}$ \\
\hline At3g50670 & Small nuclear ribonucleoprotein (U1-70k) & $3.7 * * *$ & $1.67 * * *$ & 2.90 & $2.52 * * *$ & $1.25 * *$ & $3.44 * * *$ \\
\hline At3g54640 & Tryptophan synthase, $\alpha$ subunit (TSA1) & $3.35 * * *$ & $2.46 * *$ & 3.38 & $4.61 * * *$ & 1.35 & $4.33 * *$ \\
\hline At3g57520 & Glycosyl hydrolase & $4.58 * * *$ & $1.67 *$ & $3.41 * *$ & $4.26 * * *$ & 1.53 & $3.82 * * *$ \\
\hline At4g01850 & $S$-adenosylmethionine synthase 2 & $2.41 * *$ & $2.25 * *$ & 1.82 & $3.25 * * *$ & 1.52 & $3.07 * *$ \\
\hline At4g11320 & Cysteine proteinase & $5.42 * * *$ & 1.00 & $4.37 *$ & $6.69 * * *$ & 1.00 & $6.30 * *$ \\
\hline At4g16760 & Acyl-CoA oxidase like protein & $3.02 * * *$ & $2.14^{*}$ & 3.09 & $3.21 * * *$ & $1.41 *$ & $2.85 * * *$ \\
\hline At4g26080 & Protein phosphatase ABI1 & $1.51^{* *}$ & $1.92 * * *$ & 1.85 & $2.21 * * *$ & 1.19 & $1.75^{*}$ \\
\hline At4g30530 & Glutamine amidotransferase & $4.88 * * *$ & $1.86^{* *}$ & $3.43 * *$ & $4.72 * * *$ & 1.05 & $4.00 * * *$ \\
\hline At4g31500 & Cytochrome P450 83B1 (CYP83B1) & $3.02 * * *$ & $2.98 * * *$ & $2.55 * *$ & $3.32 * * *$ & $1.67 * * *$ & $3.01 * * *$ \\
\hline At4g39950 & Cytochrome P450 79B2 (CYP79B2) & $3.67 * * *$ & $1.74 * *$ & $3.95 * *$ & $4.47 * * *$ & $1.30 * *$ & $3.52 * * *$ \\
\hline At5g05730 & Anthranilate synthase, $\alpha$ subunit (ASA1) & $3.31 * * *$ & $1.76^{*}$ & 2.63 & $3.21 * * *$ & $1.33 *$ & $3.07 * * *$ \\
\hline At5g06860 & Polygalacturonase inhibiting prot. (PGIP1) & $3.23 * *$ & 1.16 & $2.66 * * *$ & $2.37 *$ & 1.22 & 1.49 \\
\hline At5g07460 & Peptide methionine sulfoxide reductase & $1.80 * * *$ & $1.74 * *$ & 1.61 & $2.08 * * *$ & $1.24 * *$ & $1.95 * *$ \\
\hline At5g15650 & Reversibly glycosylated polypeptide-3 & $2.34 * * *$ & $1.86^{*}$ & $2.86^{*}$ & $2.83 * * *$ & $1.74 * * *$ & $2.08 * *$ \\
\hline At5g22630 & Prephenate dehydratase family & $2.57 * * *$ & $3.01 * * *$ & 2.13 & $3.74 * * *$ & 1.65 & $3.03 * *$ \\
\hline At5g24770 & Vegetative storage protein (VSP2) & $9.27 * * *$ & 1.00 & $7.12 *$ & $5.29 *$ & 1.07 & $3.79 * *$ \\
\hline At5g42650 & Allene oxide synthase (AOS) & $5.57 * * *$ & $1.25^{*}$ & $3.66^{*}$ & $3.94 * * *$ & 1.11 & $3.79 * * *$ \\
\hline At5g44320 & Translation initiation factor & $2.54 * * *$ & $1.34 *$ & 3.44 & $2.82 * * *$ & $1.11 *$ & $2.61 * * *$ \\
\hline At5g44720 & Expressed protein & $2.10 * *$ & $1.29 *$ & $1.82 * *$ & $2.18 * * *$ & 0.87 & $2.14 * * *$ \\
\hline At5g54160 & $O$-methyl transferase & $2.54 * * *$ & 1.54 & $2.51 * * *$ & $2.95 * * *$ & 1.48 & $3.19 *$ \\
\hline At5g54810 & Tryptophan synthase, $\beta$ subunit 1 (TSB1) & $2.16^{* * * *}$ & $2.48 * * *$ & $1.99 * *$ & $2.30 * * *$ & $1.18^{*}$ & $2.84 * *$ \\
\hline At5g64260 & Phi-1-related protein & $2.51 * * *$ & $2.81 * * *$ & 1.76 & $2.96 * * *$ & $1.80^{*}$ & $2.84 * *$ \\
\hline At5g64570 & Glycosyl hydrolase family 3 (XYL4) & $2.01 * * *$ & $1.58 * *$ & 2.12 & $2.38 * * *$ & $1.44^{*}$ & $1.68 *$ \\
\hline At5g67300 & Myb-related protein & $2.54 * * *$ & $2.30 * *$ & 2.08 & $3.63 * * *$ & $1.48 * *$ & $3.14 *$ \\
\hline
\end{tabular}


product ABA2 is involved in the conversion of xanthoxin to ABA-aldehyde (Gonzalez-Guzman et al. 2002), and aba3-1, a mutant in a molybdenum cofactor sulfurase required for the activation of the abscisic aldehyde oxidase (AAO3), which oxidizes ABA-aldehyde into ABA (Xiong et al. 2001). These mutants have 9 and $30 \%$, respectively, of wild-type ABA levels in turgid leaves and 2 and 10\%, respectively, of wild-type levels in partly dehydrated leaves (Leon-Kloosterziel et al. 1996). Relative changes in transcript abundance after attack by $P$. rapae were monitored and showed that 25 genes were differentially regulated between Col-0 and aba2-1 (Fig. 4A). In all, 14 genes showed a significantly higher transcript level in Col-0 than in aba2-1, including, for example, VEGETATIVE STORAGE PROTEIN2 (VSP2) and POLYGALACTURONASE INHIBITING PROTEIN1 (PGIP1), whereas 11 genes showed a significantly higher transcript level in aba2-1 than in Col-0, including PLANT DEFENSIN1.2a (PDF1.2) and LIPOXYGENASE2 (LOX2) and BETA GLUCOSIDASE1 (BG1) (Table 3). Most of these changes also were found with the aba3-1 mutant (Fig. 4C). During $S$. littoralis herbivory, a similar pattern was detected (Fig. 4B and D). Moreover, the majority of ABA-regulated genes were COI1-dependent (Table 1).

The number of transcripts whose levels change in each genotype in response to either insect is summarized in Table 4. It shows that the overlap in response to the two insects is high and that the response of the plant generally was similar, with the exceptions described above. Several genes were still upregulated by herbivory in all mutants tested, indicating that other signals must be involved to control these genes. A dehydration treatment was performed and showed that water loss increased the transcript abundance of some of these genes. Thus, the abiotic stress caused by insect feeding is likely to generate signals regulating these genes.

\section{Conserved transcript profile after $5 \mathrm{~h}$ and 8 days.}

To test whether genes present on the dedicated microarray also were upregulated in the later phase of herbivory, the transcript profile observed after $5 \mathrm{~h}$ of feeding by third- and fourthinstar $S$. littoralis larvae were compared with the transcript profile after 8 days of constant feeding. In this experiment, newly hatched $S$. littoralis larvae were placed on Arabidopsis and left to feed for 8 days. Approximately $90 \%$ of genes upregulated after $5 \mathrm{~h}$ also showed a higher transcript level after 8 days (Fig. 5). A few RNAs were slightly more abundant after 8 days (for example, VSP2 and $O P R 3$ ) but, overall, the transcript signature was strikingly similar. Relative transcript levels also were measured in aba2-1 after 8 days of herbivory. A majority of ABA-regulated genes showed a similar expression profile after $5 \mathrm{~h}$ and 8 days of feeding (Table 3 ). These data indicate that the dedicated microarray was not biased with early responsive genes because they were equally regulated by young and more mature larvae, either during a short and intense damage or during a slow and continuous feeding over many days.

\section{Insect performance on signaling mutants.}

The specific roles of most genes in the direct defense against chewing herbivores are still unknown. Recently, it was shown that $P$. rapae larvae feeding on coil-1 plants were heavier than larvae feeding on wild-type plants (Reymond et al. 2004). This effect was relatively small but significant and was attributed to the lack of induction of the jasmonate-dependent defense genes. Larval performance of S. littoralis was evaluated on wild-type and coil-1 plants to test whether induced defense genes also contributed to plant resistance against this generalist herbivore. Thirty-three newly hatched larvae were placed on each Arabidopsis genotype and the weight of the larvae was measured after 8 days. $S$. littoralis larvae gained significantly more weight on coil-1 plants than on Col-0 plants (Fig. 6). Interestingly, this difference was less marked with $P$. rapae. In nine independent experiments, $S$. littoralis larvae gained, on average, $182 \pm 30 \%$ more weight on coil-1 plants than on Col0 . In contrast, the relative increase of $P$. rapae weight was $39 \pm$ $11 \%$ in four independent experiments. In addition, leaf damage was always more extensive on coil-1 than on Col-0 plants after feeding by both species (data not shown).

Having observed a much larger difference in weight gain with $S$. littoralis than with $P$. rapae larvae between Col-0 and coil-1 plants, larval performance was tested on other Arabidopsis signaling mutants with $S$. littoralis, reasoning that intermediate performance might be easier to detect with $S$. littoralis than with $P$. rapae. First, $S$. littoralis larvae were significantly heavier when feeding on aba2-1 mutants than when feeding on

Table 2. Representative salicylic acid (SA)-regulated genes in response to Pieris rapae and Spodoptera littoralis feeding ${ }^{\mathrm{a}}$

\begin{tabular}{|c|c|c|c|c|c|c|c|c|}
\hline \multirow[b]{2}{*}{ AGI } & \multirow[b]{2}{*}{ Description } & \multicolumn{3}{|c|}{ Pieris rapae } & \multicolumn{4}{|c|}{ Spodoptera littoralis } \\
\hline & & Col-0 & sid2-1 & npr1-1 & Col-0 & sid2-1 & npr1-1 & COI-dep. ${ }^{b}$ \\
\hline Atlg02920 & Glutathione $S$-transferase (GST11/GSTF7) & $2.39 * * *$ & $2.11 *$ & $1.81^{*}$ & $3.35 * * *$ & $2.46 * *$ & $1.98 *$ & \\
\hline At1g09070 & Similar to cold-regulated gene SRC2 & $2.13^{*}$ & $2.38 *$ & 1.89 & $3.80 * * *$ & $1.61 *$ & 1.29 & \\
\hline At $\lg 17420$ & Lipoxygenase (LOX3) & $7.79 * * *$ & $6.64 * *$ & $4.13^{*}$ & $14.43 * * *$ & $4.19 * *$ & $4.08 *$ & Yes \\
\hline At1g27730 & Salt-tolerance zinc finger protein & $1.71 *$ & 1.88 & 2.13 & $4.84 * *$ & 1.35 & 1.39 & Yes sup \\
\hline At1g45145 & Thioredoxin H-type 5 (TRX-H-5) & $1.24 * *$ & $1.18 * * *$ & $1.15^{*}$ & $2.16^{* * *}$ & $1.17 *$ & $1.32 * *$ & Yes \\
\hline At1g52890 & No apical meristem (NAM) protein & $2.27 * * *$ & $2.10 * * *$ & $2.10 * *$ & $5.02 * * *$ & $1.89 * * *$ & $1.85 * *$ & Yes \\
\hline At1g74100 & Desulfoglucosinolate sulfotransferase & $2.29 * * *$ & $2.33 * * *$ & $2.08 * * *$ & $3.21 * * *$ & $2.13 * * *$ & $1.82 * *$ & Yes \\
\hline At $2 \mathrm{~g} 22500$ & Mitochondrial carrier protein family & $1.51^{*}$ & $1.47 * *$ & 1.53 & $3.06 * * *$ & 1.16 & 1.04 & Yes sup \\
\hline At $2 \mathrm{~g} 37180$ & Aquaporin PIP2.3 (RD28) & $3.54 * * *$ & $2.40 * * *$ & $2.50 * *$ & $3.62 * * *$ & $1.88 * *$ & $2.26 * * *$ & Yes \\
\hline At $2 \mathrm{~g} 41100$ & Calmodulin-related protein $3(\mathrm{TCH} 3)$ & 1.61 & 1.53 & 1.74 & $2.90 * *$ & 1.54 & 1.21 & Yes sup \\
\hline At3g22600 & Protease inhibitor/seed storage/LTP & $1.89 *$ & 1.40 & $1.93 *$ & $2.74 * * *$ & 1.30 & 1.36 & \\
\hline At3g44260 & CCR4-associated factor 1-related & $2.46^{*}$ & 2.11 & 1.91 & $4.72 * *$ & 1.61 & 1.39 & \\
\hline At3g52800 & Zinc finger -like protein & $1.97 * *$ & $2.02 *$ & 1.44 & $2.55 * * *$ & $1.42 *$ & $1.37 *$ & \\
\hline At3g55980 & Zinc finger transcription factor (PEI1) & 1.31 & 1.52 & 1.35 & $2.96^{*}$ & 1.22 & 1.02 & Yes sup \\
\hline At4g02380 & LEA protein family & $1.59 *$ & $1.87 *$ & 1.06 & $4.53 * *$ & $1.57 *$ & 1.03 & Yes sup \\
\hline At4g26080 & Protein phosphatase ABI1 & $1.51 * *$ & $1.74 * *$ & $1.22 *$ & $2.21 * * *$ & $1.37 * *$ & $1.35 *$ & Yes \\
\hline At $4 \mathrm{~g} 30530$ & Glutamine amidotransferase & $4.88 * * *$ & $3.64 * * *$ & $3.75 * * *$ & $4.72 * * *$ & $3.67 * * *$ & $3.66 * * *$ & Yes \\
\hline At5g06320 & Harpin-induced protein 1 family & 1.25 & $1.35^{*}$ & $1.45^{*}$ & $2.60 * * *$ & 1.14 & 1.10 & Yes sup \\
\hline At5g37770 & Calmodulin-related protein $2(\mathrm{TCH} 2)$ & 1.99 & $2.29 * *$ & 1.24 & $4.36 * *$ & 1.33 & 0.90 & \\
\hline At5g67300 & Myb-related protein (MYB44) & $2.54 * * *$ & $2.28 * *$ & $2.57 * *$ & $3.63 * * *$ & $2.05 * *$ & $1.96^{*}$ & Yes \\
\hline
\end{tabular}

${ }^{a}$ Expression ratios are color coded according to intensity: yellow (from 2 to 4 ), light orange (from 4 to 6 ), dark orange (from 6 to 10 ), red ( $>10$ ).

${ }^{\mathrm{b}}$ Genes suppressed by COI1 (Yes sup) or dependent on COI1 (Yes) in response to $P$. rapae or $S$. littoralis are indicated.* $\mathrm{P}$ value $<0.05$, ** $\mathrm{P}$ value $<0.01$,

*** $\mathrm{P}$ value $<0.001$ ( $\mathrm{P}$ values were calculated on log-transformed ratios from treatment and control experiments, Student's $t$-test). 
Col-0, although the difference was not as large as with coil-1 plants (Fig. 7A). In three independent experiments, larvae gained $70 \% \pm 30 \%$ more weight on the aba2-1 mutant. The mutant $a b a 3-1$ yielded mixed results: in some replicates, larvae performed better on the mutant whereas, in other replicates, there was no significant difference, which could be explained by the 10 to $30 \%$ wild-type ABA levels that are still present in this mutant (Leon-Kloosterziel et al. 1996) (data not shown). With the SA-signaling mutants sid2-1 and nprl-1, or with the ET-signaling mutant ein2-1, the effect was opposite to coil-1; after 8 days of feeding, larvae were significantly smaller on all mutants than on Col-0 plants (Fig. 7B and C). These observations were confirmed in three independent experiments for
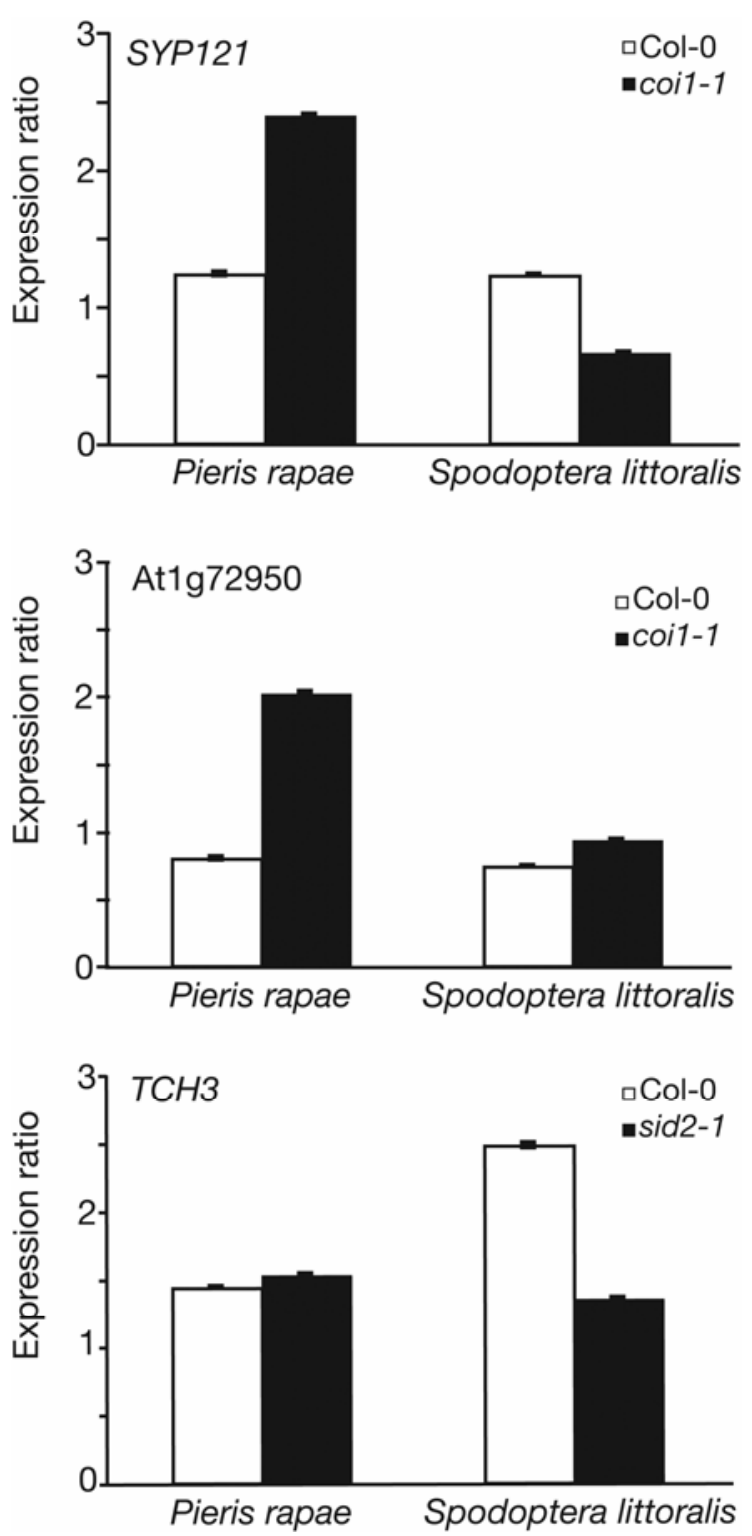

Fig. 3. Real-time quantitative polymerase chain reaction analysis of selected genes differentially regulated in response to Spodoptera littoralis or Pieris rapae feeding. Expression levels of SYP121 (At3g13920) and of a gene encoding a disease resistance protein (toll interleukin 1 receptornucleotide binding site class) (At1g72950) were measured in Col-0 and coil-1 plants, and expression levels of TCH3 (At2g41100) were measured in Col-0 and sid2-1 plants after $5 \mathrm{~h}$ of feeding. Expression levels were normalized with respect to the housekeeping gene EIF4A1 (At3g13920) and expression ratios were calculated by dividing the expression levels in infested plants with the expression levels in control plants. Data bars represent the mean ( \pm standard error) of three technical repeats. This experiment was repeated once with similar results. each genotype; larvae had smaller masses on sid2 (39 $\pm 17 \%)$, $n p r 1-1(39 \pm 3 \%)$, and ein $2-1(34 \% \pm 5 \%)$ plants.

\section{DISCUSSION}

JA, ET, and SA are global regulators for defense gene expression during bacterial and fungal pathogenesis. A goal of this study was to elucidate the role of these signals in the control of transcripts levels during herbivory in Arabidopsis. In addition, the implication of these regulators in the response to two insect species of different diet breadth, the specialist $P$. $\mathrm{ra}$ pae and the generalist $S$. littoralis, was tested. Using a dedicated microarray containing insect- and defense-regulated genes, it was shown first with the coil-1 mutant that the JA pathway was required for the upregulation of a similar set of genes after $P$. rapae and $S$. littoralis herbivory, highlighting the importance of this signal in the control of transcript abundance after herbivory by both a specialist and a generalist insect. On average, changes in RNA abundance of more than half of the insect-regulated genes present on the microarray depended on a functional COI1 protein. Using a nearly full genome Arabidopsis microarray, we recently found that approximately $60 \%$ of $P$. rapae-upregulated genes showed no change in transcript level in coil-1 plants, confirming the results obtained with our dedicated microarray (data not shown).

Interestingly, $P$. rapae larvae feeding on coil-1 plants increased the transcript levels of a set of genes that normally are not induced in Col-0 plants, illustrating a potential suppression of some defense genes by $P$. rapae in wild-type plants. Among those were genes that clearly have been shown to play a role in the defense against pathogenic fungi and bacteria. They included, for example, a disease resistance gene (TIR-NBS class), $N P R 1$, which is a key nuclear regulator of the induction of SA-dependent $P R$ genes, two $P R$ genes ( $P R 5$ and class IV chitinase $C H I V)$, a mitogen-activated protein kinase $(M P K 3)$ that is upregulated by fungal-cell-wall-derived chitin oligomers (Zhang et al. 2002), a glutathione-S transferase (GST6/GSTF8) that accumulates after infection with Pseudomonas syringae (Jones et al. 2004; Varet et al. 2002), and a gene encoding a syntaxin $(P E N 1 / S Y P 121)$ that is required for resistance against powdery mildew (Collins et al. 2003). Surprisingly, the JA-dependent suppression of induction was specific to Pieris rapae feeding, because transcript levels of these genes either were not changed or increased similarly in response to $S$. littoralis feeding in both wild-type and coil-1 plants.

One aspect of the evolutionary arms race between plants and herbivores has been illustrated recently by the finding that insects suppress induced defenses of the plant. For example, $M$. sexta suppressed nicotine production in $N$. tabacum (Kahl et al. 2000). Glucose oxidase has been identified in the saliva from Helicoverpa zea as the factor responsible for a decrease in nicotine production (Musser et al. 2002). Silverleaf whitefly repressed JA-regulated defenses to allow a better nymph development (Zarate et al. 2007). This study provides another example of insect-triggered suppression of defenses and shows that this phenomenon depends on COI1. Curiously, the genes that are under suppression are likely to be involved in resistance to bacterial or fungal pathogens. At present, there is no obvious explanation for this observation; however, there is growing evidence that plant defenses against herbivores and bacterial pathogens are interconnected. For example, $P$. rapae herbivory enhanced resistance to the pathogens Pseudomonas syringae and Xanthomonas campestris (De Vos et al. 2006). In contrast, $P$. syringae-infected plants were more resistant to $T$. $n i$ (Cui et al. 2005). Bacteria also are able to interfere with the host defense system by suppressing the induction of defense genes. Some strains of $P$. syringae produce the phytotoxin coronatine, 
a functional analog of JA, which is required for full virulence (Kloek et al. 2001; Zhao et al. 2003). Thus, insects and bacteria manipulate plant defense and, intriguingly, this effect is mediated through COI1 in both cases. Because the increase in transcript abundance of some PR genes was suppressed by Pieris rapae but not by $S$. littoralis, it would be interesting to compare resistance to bacterial pathogens after feeding by these different insects. It also would be important to test whether $P$. rapae regurgitant contains a suppressive molecule that is not present in $S$. littoralis regurgitant.

Specialist herbivores have been predicted to be more tolerant to defense responses of their host compared with generalists (Bernays 1998). The present study shows that the generalist herbivore $S$. littoralis was more susceptible to the induced defenses of the plant than the specialist $P$. rapae. Indeed, $S$. littoralis larvae were, on average, three times heavier on the de- fense-compromised mutant coil-1 than on Col-0, whereas $P$. rapae larvae gained only $38 \%$ more weight, indicating that genes showing no change in transcript level in coil-1 in response to these insects might be important for defense in wildtype plants and illustrating a better tolerance of $P$. rapae to the plant defense system. This argues in favor of an adaptation of the insects to the plant toxic chemistry rather than the plants inducing different defense genes in response to different chewing herbivores. This was illustrated by the observation that the specialist grew better on wild-type plants than the generalist. In other words, the success of specialist herbivores compared with generalists may depend more on potent detoxification or tolerance machinery rather than on the manipulation of the host defense gene expression. One recent example is the discovery that $P$. rapae larvae are adapted to the glucosinolatemyrosinase complex and contain a nitrile-specifier gut protein
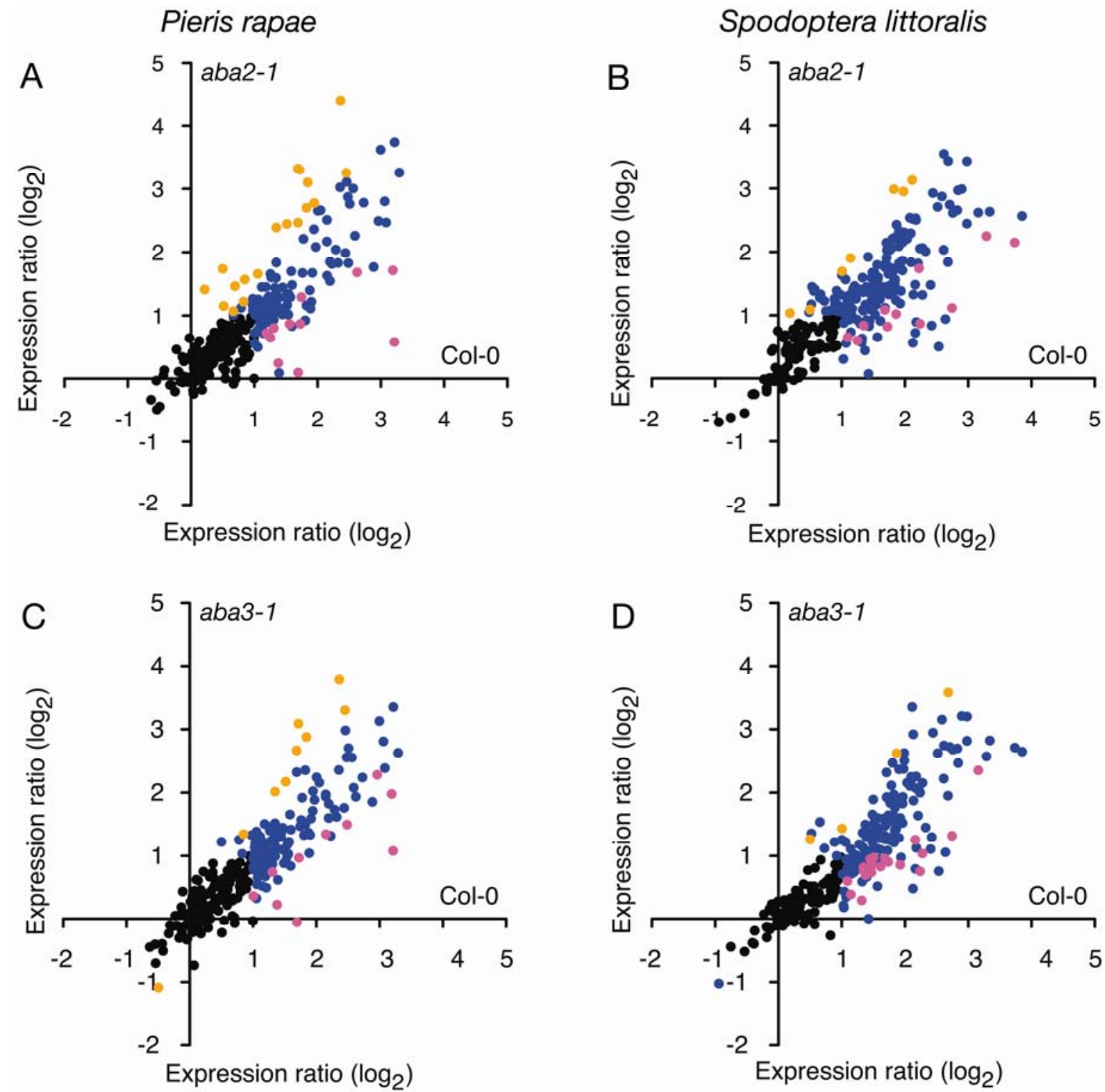

Fig. 4. Changes in transcript level in wild-type and mutant Arabidopsis plants after challenge for $5 \mathrm{~h}$ with $\mathbf{A}$ and $\mathbf{C}$, Pieris rapae and $\mathbf{B}$ and $\mathbf{D}$, Spodoptera littoralis. Relative changes in RNA abundance after challenge with insects were measured in Col-0 in different signaling mutant plants: A and B, aba2-1 and $\mathbf{C}$ and $\mathbf{D}, a b a 3-1$. Mean expression ratios calculated from experiments comparing challenged and unchallenged Col-0 plants (eight independent replicates for $P$. rapae and six independent replicates for $S$. littoralis) are plotted against mean expression ratios between challenged and unchallenged mutant plants (four independent replicates). Black dots represent genes that showed no changes in transcript abundance, blue dots represent genes that showed a higher transcript level in both genotypes in response to herbivory but with no statistical difference between genotypes, magenta dots represent genes that showed a significantly larger change in transcript level in wild-type than in mutant plants, and orange dots represent genes that showed a significantly larger change in transcript level in mutant than in wild-type plants (Student's $t$ test). 
that, upon hydrolysis of glucosinolates by myrosinases during insect feeding, allows the formation of nitriles instead of the more toxic isothiocyanates (Wittstock et al. 2004). Strikingly, the authors found that this enzymatic activity is absent in S. littoralis larvae.

An alternative explanation for the smaller effect of coil-1 on $P$. rapae might be that the presence of defenses that are expressed in the absence of JA perception counterbalance those that are no longer induced in the mutant, resulting in an apparent weak effect of the mutation. A whole-genome analysis of coil-1 plants in response to $P$. rapae and S. littoralis might reveal defense genes that are upregulated only by the specialist herbivore and that play a direct role against larval growth.

To assess the importance of ET and SA pathways, transcript profiles of ein2-1, sid2-1, and nprl-1 mutants after $P$. rapae and $S$. littoralis herbivory were compared. In the case of $P$. rapae, these signals appear not to play a major role in the regulation of the genes present on the array because the expression profiles in the mutants were almost identical to Col-0 plants. It was shown recently that feeding by $P$. rapae larvae induces a slow and modest increase in ET production (De Vos et al. 2005). In the same study, no SA accumulation was found after $P$. rapae attack. This markedly contrasts with the fast and significant increase of JA production that is observed during herbivory (De Vos et al. 2005; McCloud and Baldwin 1997; Reymond et al. 2004). Hence, the transcript profiling of Col-0 and coil-1, sid2-1, and ein2-1 mutants corroborates the signal signatures of $P$. rapae-attacked Arabidopsis plants.

However, in the case of $S$. littoralis, the transcript profiles in the mutants were different from those observed in Col-0 plants. Both SA and ET were found to control gene expression. For example, some genes that depended on SID2 and NPR1 had twofold higher transcript levels after $S$. littoralis feeding than after $P$. rapae herbivory, including $L O X 3$ and an NAC transcription factor (At1g52890). To test whether the signal signature was different in the case of $S$. littoralis feeding, SA was measured in wild-type plants after feeding of the two species of insects and no significant increase was observed, ruling out a higher SA accumulation after S. littoralis herbivory (N. Bodenhausen, P. Duechting, and E. Weiler, personal communication).

Interestingly, a majority of SID2/NPR1-dependent genes were regulated by COI1. Some genes that showed a smaller change in transcript abundance in sid2-1 or nprl-1 compared with Col-0 in response to $S$. littoralis also were dependent on COI1. Moreover, other SID2/NPR1-dependent genes were

Table 4. Total number of genes induced in each genotype in response to either Pieris rapae or Spodoptera littoralis ${ }^{\mathrm{a}}$

\begin{tabular}{lccc}
\hline & \multicolumn{2}{c}{ Induced by } & \\
\cline { 2 - 3 } Genotype & P. rapae & S. littoralis & Overlap \\
\hline Col-0 & 82 & 117 & 104 \\
coi1-1 & 91 & 29 & 51 \\
ein2-1 & 57 & 67 & 68 \\
sid2-1 & 77 & 62 & 76 \\
npr1-1 & 64 & 65 & 71 \\
aba2-1 & 75 & 106 & 97 \\
aba3-1 & 62 & 76 & 74 \\
\hline
\end{tabular}

a The number of genes up-regulated by one insect include genes with an expression ratio $>2(P<0.05)$. Genes considered as up-regulated by both insects (overlap) include genes with an expression ratio $>2(P<0.05)$ in response to one insect and at least $>1.5(P<0.05)$ in response to the other insect.

Table 3. Representative ABA-regulated genes in response to Pieris rapae and Spodoptera littoralis feeding ${ }^{\mathrm{a}}$

\begin{tabular}{|c|c|c|c|c|c|c|c|c|}
\hline \multirow[b]{3}{*}{ AGI } & \multirow[b]{3}{*}{ Description } & \multirow{2}{*}{\multicolumn{2}{|c|}{$\begin{array}{c}\text { Pieris rapae } \\
5 \mathrm{~h}\end{array}$}} & \multicolumn{5}{|c|}{ Spodoptera littoralis } \\
\hline & & & & \multicolumn{3}{|c|}{$\mathbf{5} \mathbf{h}$} & \multicolumn{2}{|c|}{8 days } \\
\hline & & Col-0 & $a b a 2-1$ & Col-0 & $a b a 2-1$ & Col-0 & $a b a 2-1$ & $\mathrm{COI}^{\mathrm{b}}$ \\
\hline \multicolumn{9}{|c|}{ Positive regulation by $\mathrm{ABA}$} \\
\hline At1g02920 & Glutathione $S$-transferase (GST11/GSTF7) & $2.39 * * *$ & 1.57 & $3.35 * * *$ & $2.51 * * *$ & $3.70 * * *$ & 1.40 & No \\
\hline At1g02930 & Glutathione $S$-transferase (GST1/GSTF6) & $2.90 * *$ & 2.02 & $4.67 * * *$ & $3.34 * * *$ & $4.73 *$ & 1.39 & No \\
\hline At1g09970 & LRR transmembrane protein kinase & $2.48 * * *$ & $1.75^{* *}$ & $3.21 * * *$ & $2.17 *$ & $3.93 * * *$ & 1.77 & No \\
\hline At1g74100 & Desulfoglucosinolate sulfotransferase & $2.29 * * *$ & $1.98 * * *$ & $3.21 * * *$ & $2.12 * * *$ & $2.99 * * *$ & 1.36 & Yes \\
\hline At1g78850 & Curculin-like (mannose-binding) lectin & $2.60 * * *$ & 1.19 & $2.36 * * *$ & $1.91 * *$ & $2.59 * *$ & 1.70 & No \\
\hline At2g36830 & Major intrinsic protein family $(\gamma$-TIP) & $2.94 * * *$ & $1.83 *$ & $2.63 * *$ & 2.21 & 2.28 & 1.64 & Yes \\
\hline At2g47180 & Galactinol synthase & $1.73^{* *}$ & $1.78 * *$ & $3.30 * * *$ & $1.77 * *$ & $5.89 * * *$ & 2.73 & Yes \\
\hline At3g44260 & CCR4-NOT transcription complex protein & $2.46^{*}$ & $2.46^{*}$ & $4.72 * *$ & $1.82 *$ & $3.41 * *$ & 1.65 & No \\
\hline At3g54640 & Tryptophan synthase, alpha subunit (TSA1) & $3.35 * * *$ & $2.45 * * *$ & $4.61 * * *$ & $3.53 * * *$ & $4.53 * *$ & 1.54 & Yes \\
\hline At4g11320 & Cysteine proteinase & $5.42 * * *$ & $3.97 * *$ & $6.69 * * *$ & $2.17 *$ & $10.51 * * *$ & 5.02 & Yes \\
\hline At4g15440 & Hydroperoxide lyase (CYP74B2/HPL1) & $3.74 * * *$ & $2.33^{*}$ & $3.63 * * *$ & $2.03 *$ & $2.76^{*}$ & 2.64 & Yes \\
\hline At5g05730 & Anthranilate synthase, alpha subunit (ASA1) & $3.31 * * *$ & $1.83 * * *$ & $3.21 * * *$ & $2.73 * * *$ & $4.78 * * *$ & 2.13 & Yes \\
\hline At5g06860 & Polygalacturonase inhibiting protein (PGIP1) & $3.23 * *$ & 1.07 & $2.37 *$ & $1.91 *$ & $2.28 * * *$ & 1.90 & Yes \\
\hline At5g24770 & Vegetative storage protein (VSP2) & $9.27 * * *$ & 1.50 & $5.29 *$ & 2.79 & $31.77 * * *$ & 7.83 & Yes \\
\hline At5g64570 & Glycosyl hydrolase family 3 (XYL4) & $2.01 * * *$ & $1.48 * *$ & $2.38 * * *$ & $1.52 * *$ & $3.17 * * *$ & 1.66 & Yes \\
\hline \multicolumn{9}{|c|}{ Negative regulation by $\mathrm{ABA}$} \\
\hline At1g52400 & ABA-glucose hydrolase (BG1) & $5.14 * * *$ & $21.07 * * *$ & $4.33 * * *$ & $8.78 * * *$ & $12.47 * *$ & 23.45 & Yes \\
\hline At1g54010 & Myrosinase-associated protein & $3.29 *$ & $9.86 * * *$ & $5.72 * *$ & $6.55 * * *$ & $4.85 *$ & 5.66 & Yes \\
\hline At $2 \mathrm{~g} 24850$ & Tyrosine aminotransferase (TAT3) & $3.59 * * *$ & $8.58 * * *$ & $6.13 * * *$ & $11.71 * * *$ & $7.42 * * *$ & 5.66 & Yes \\
\hline At2g37040 & Phenylalanine ammonia lyase (PAL1) & $2.08 * * *$ & $3.16^{* * *}$ & $4.28 * * *$ & $3.57 * *$ & $3.88 * *$ & 3.96 & No \\
\hline At3g16410 & Myrosinase-binding protein & $5.47 * * *$ & $9.53 * * *$ & $5.99 * * *$ & $7.34 * * *$ & $9.40 * * *$ & 9.60 & Yes \\
\hline At3g45140 & Lipoxygenase (LOX2) & $3.22 * *$ & $9.99 * * *$ & $3.54 * *$ & $7.93 * * *$ & $5.35 * *$ & 8.56 & Yes \\
\hline At5g07460 & Peptide sulfoxide methionine reductase & $1.80 * * *$ & $2.97 * * *$ & $2.08 * * *$ & $2.96 * * *$ & $2.32 * * *$ & 2.10 & Yes \\
\hline At5g15970 & Cold-regulated protein (COR6.6) & 1.16 & $2.66 * *$ & $1.46^{*}$ & $1.77 * *$ & 3.31 & 2.90 & No \\
\hline At5g42650 & Allene oxide synthase (CYP74A, AOS) & $5.57 * * *$ & $7.32 * * *$ & $3.94 * * *$ & $7.74 * * *$ & $6.90 * * *$ & 9.69 & Yes \\
\hline At5g44420 & Plant defensin protein (PDF1.2a) & 1.41 & $3.34 *$ & 1.41 & $2.13 *$ & 1.27 & 5.65 & No \\
\hline
\end{tabular}

${ }^{a}$ Expression ratio are color coded according to intensity: yellow (from 2 to 4 ), light orange (from 4 to 6 ), dark orange (from 6 to 10 ), red ( $>10$ ).

${ }^{\mathrm{b}}$ Yes and No indicate whether genes are dependent on COI1 in response to P. rapae or S. littoralis. $* \mathrm{P}$ value $<0.05, * * \mathrm{P}$ value $<0.01, * * * \mathrm{P}$ value $<0.001$ ( $\mathrm{P}$ values were calculated on log-transformed ratios from treatment and control experiments, Student's $t$-test). No $\mathrm{P}$ values were calculated for the experiment 8 days aba2-1 (only two biological replicates). 
suppressed by COI1 in response to $P$. rapae (Table 2). These findings support the idea that JA and SA cooperate to activate or suppress gene expression during herbivory. Negative crosstalk between pathways has emerged recently as an important element in the defense strategies of the plant. Several studies have reported that the activation of SA-dependent resistance suppresses JA signaling in plants (Felton and Korth 2000). Exogenous treatments and genetic evidence have revealed an antagonistic effect of SA on JA-inducible changes in transcript abundance and resistance to insects (Cipollini et al. 2004;

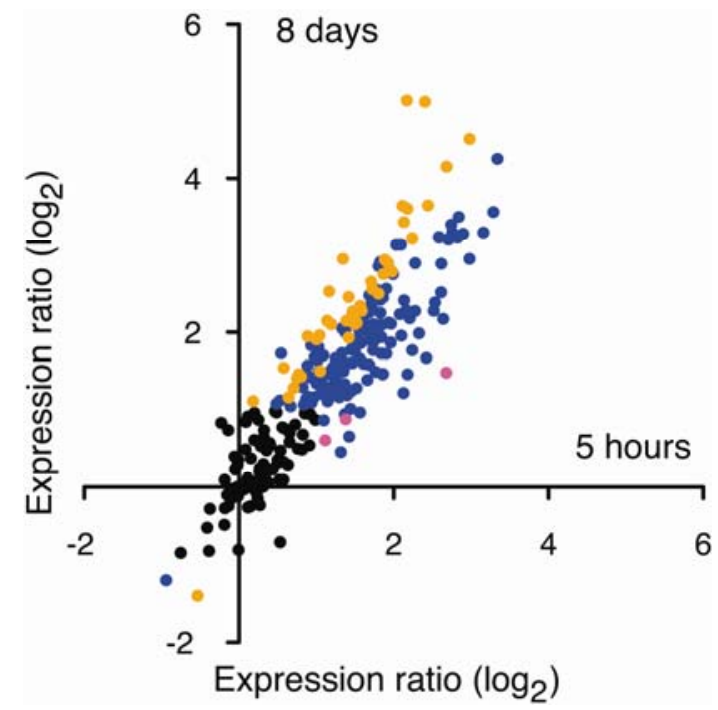

Fig. 5. Changes in transcript level in wild-type Arabidopsis plants after challenge for 5 hours or 8 days with Spodoptera littoralis. Mean expression ratios calculated from experiments comparing challenged and unchallenged after $5 \mathrm{~h}$ of feeding (six independent replicates) are plotted against mean expression ratios between challenged and unchallenged mutant plants after 8 days of feeding (five independent replicates). Three third- or fourth-instar larvae per plant were used for the 5-h treatment, compared with one to two newly hatched larvae per plant for the 8-day treatment. Black dots represent genes that showed no changes in transcript abundance, blue dots represent genes that showed a higher transcript level in both genotypes in response to herbivory but with no statistical difference between genotypes, magenta dots represent genes that showed a significantly larger change in transcript level in wild-type than in mutant plants, and orange dots represent genes that showed a significantly larger change in transcript level in mutant than in wild-type plants (Student's $t$ test).

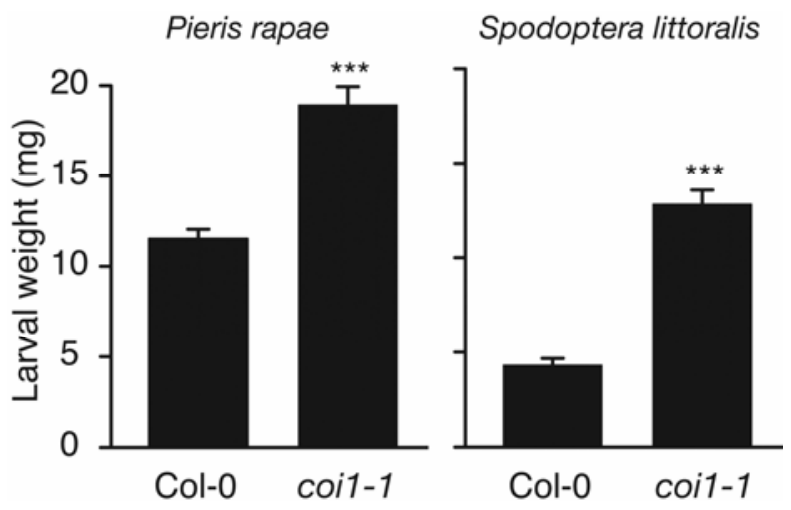

Fig. 6. Influence of the jasmonate pathway on larval performance of two lepidopteran species. Growth of the specialist Pieris rapae and the generalist Spodoptera littoralis was tested on Col-0 and coil-1 mutant plants. Freshly hatched $P$. rapae or $S$. littoralis larvae were placed simultaneously on each Arabidopsis genoytpe and larval weight (mean \pm standard error) was measured after 8 days of feeding. Asterisks indicate a significant difference at $P<0.001$ (Student's $t$ test.). This experiment was repeated nine times for $S$. littoralis and four times for $P$. rapae with similar results.
Doares et al. 1995; Gupta et al. 2000). Studies on phloemfeeding insects has led to the "decoy hypothesis" that aphids manipulate plant defense, whereby induction of the SA pathway causes a suppression of the biologically effective JA pathway (Thompson and Goggin 2006; Zhu-Salzman et al. 2005). In line with this hypothesis, it was shown recently, using a combination of Arabidopsis transcript profiling analysis and bioassays on mutants impaired in JA- and SA-signaling pathways, that silverleaf whiteflies induce SA-signaling pathways and that this results in the suppression of basal JA-dependent defenses (Kempema et al. 2007; Zarate et al. 2007). Based on these studies, it was expected that JA-inducible genes would
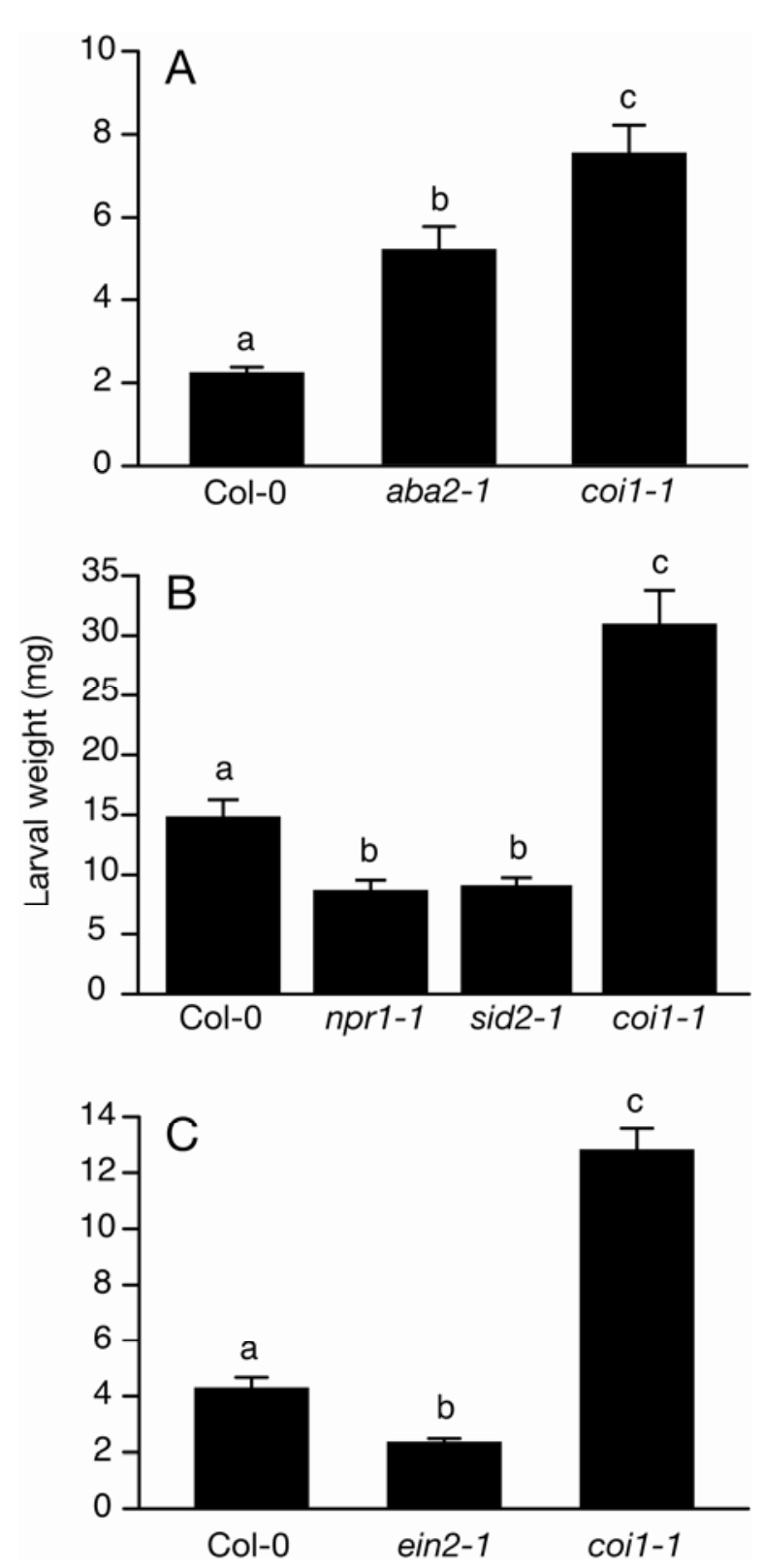

Fig. 7. Influence of signaling pathways on Spodoptera littoralis larval performance. Growth of S. littoralis was tested on A, abscisic acid-signaling mutant (aba2-1), B, salicylic acid-signaling mutants (sid2-1 and npr1-1), and $\mathbf{C}$, ethylene-signaling mutant (ein2-1). In each independent experiment, wild-type (Col-0) and jasmonic acid-signaling mutant (coil-1) plants were used as references. Freshly hatched $S$. littoralis larvae were placed simultaneously on each Arabidopsis genotype and larval weight (mean \pm standard error) was measured after $\mathbf{A}$, seven or $\mathbf{B}$ and $\mathbf{C}$, eight days of feeding. Bars with different letters differed at $P<0.05$ (Tukey's highly significant difference test). This experiment was repeated three times for each genotype with similar results. 
show a much higher transcript level in the SA mutants. In contrast, JA-dependent genes also were SA dependent. This new finding brings a new twist to the crosstalk model and indicates that, in this particular case, JA and SA might have a synergistic effect on the RNA abundance of some transcripts. However, the activation of SA-dependent responses is found in coil-1, supporting the cross-talk model. This study shows that signaling of plant-insect interactions is complex and does not necessary follow the patterns observed in plant-pathogen interactions.

Arabidopsis mutants defective in ET and SA signaling pathways both were more resistant to $S$. littoralis, indicating that both pathways negatively control insect resistance. Although the effect was marginal compared with the large increase of weight on coil-1 plants, $S$. littoralis larvae gained significantly less weight on ein2-1, npr 1-1, and sid2-1 mutant plants in several independent experiments. This is in accordance with findings obtained with the other generalists, $T$. ni and S. exigua, on SA and ET mutants (Cui et al. 2002; Mewis et al. 2005) and with observations on ein2-1 leaf consumption by $S$. littoralis (Stotz et al. 2000). The microarray experiments did not reveal defense genes with higher transcript levels in either SA or ET mutants after feeding with S. littoralis; therefore, the SA and ET pathways might negatively control insect resistance through other mechanisms, such as changes in constitutive levels of defense compounds or steps that are not correlated with changes in RNA abundance. Alternatively, reciprocally regulated JA or SA genes might have been missed due to the strategy that was chosen to design the microarray.

In Arabidopsis, wounding activates the ET and JA pathways and the cooperation of both signals is proposed to simultaneously trigger the activation of wounding-responsive genes through the JA-dependent transcription factor MYC2 and to repress wounding-responsive genes through the ET-dependent transcription factor ERF1 (Lorenzo et al. 2004). Thus, the finding that ein2-1 is more resistant to $S$. littoralis feeding also could be explained by the lack of repression of wound-responsive genes. A whole-genome microarray study of insect-challenged ein2-1 plants will be necessary to resolve this hypothesis.

Searching for other signals that could regulate transcript levels in response to herbivory, a moderate but consistent and similar alteration in transcript profiles was observed in two mutants in the ABA biosynthetic pathway in response to the two insects tested, indicating that this hormone contributes significantly to the observed changes in RNAs abundance. In addition, the aba2-1 mutant impaired in ABA synthesis allowed a larger weight gain of $S$. littoralis larvae than Col-0 plants. ABA has been implicated in the response to abiotic stress such as drought or wounding (Christmann et al. 2006) and was shown to control S. exigua feeding in tomato (Thaler and Bostock 2004), but there was, as yet, no direct evidence for its implication in the response to herbivory in Arabidopsis.

Of the ABA-regulated genes, VSP2, which had a much lower transcript level by $S$. littoralis in aba2-1, recently was shown to encode an acid phosphatase that delays the development of the cowpea bruchid Callosobruchus maculatus and of Drosophila melanogaster, and increases the mortality of the southern corn rootworm Diabrotica undecimpunctata howardi when incorporated into the diet (Liu et al. 2005). The PGIPI gene encoding a polygalacturonase-inhibiting protein also was not upregulated in aba2-1. Polygalacturonases have been identified in some phytophagous insects and are thought to be involved in the maceration of plant tissue during feeding (Girard and Jouanin 1999; Shen et al. 1996). Moreover, PGIPs from bean were found to inhibit insect polygalacturonases in vitro (D'Ovidio et al. 2004). In addition, several genes encoding enzymes in the tryptophan synthesis pathway (ASA1, TSA1) and the indole glucosinolate pathway (desulfoglucosinolate trans- ferase) showed lower transcript levels in the two aba mutants. Hence, a smaller change in RNA abundance of all these defense genes in these mutants could have an additive effect and diminish the plant resistance to feeding larvae.

ABA had both a positive and a negative regulatory effect on transcript levels. Indeed, some genes, such as VSP2, showed a smaller change in RNA abundance in response to $P$. rapae and $S$. littoralis in aba mutants, whereas some genes, such as $P D F 1.2$, had an opposite pattern in the mutants. It is noteworthy that most of the ABA-regulated genes were known markers of the JA pathway and were found to be COI1-dependent. Recently, an antagonistic interaction between the ABA and the JA and ET pathways was reported (Anderson et al. 2004). In their study, the authors proposed a model in which ABA negatively regulates pathogen-responsive genes such as $P D F 1.2$ and positively regulates wound-responsive genes such as $V S P 2$, in both cases via the JA pathway. Interestingly, the same antagonistic effect of ABA on insect-regulated genes was observed in the present study and this could illustrate how, during herbivory, the plant selects the appropriate defense response.

An extension of this study on the whole Arabidopsis genome would give further insight into the exact quantitative contribution of defense-signaling pathways in the relative changes in RNA abundance triggered by herbivory. Recent transcriptome analyses of Arabidopsis plants subjected to herbivorous insects with different modes of attack revealed a relatively small overlap between a chewing herbivore, a cell-content-feeding thrips, a phloem-feeding aphid, and a phloem-feeding whitefly (De Vos et al. 2005; Kempema et al. 2007). Thus, the role of different signaling pathways might differ depending on the plantattacker combination.

In conclusion, this study shows that insect attack triggers changes in transcript levels that are controlled mainly by JA and, to a lesser extent, by ABA, SA, and ET. It also provides genetic evidence that JA and ABA pathways significantly contribute to resistance to insects. Uncovering the functional role of individual insect-regulated genes in the response to herbivores will be a challenge for future research and might help generate knowledge to develop new strategies for crop protection.

\section{MATERIALS AND METHODS}

\section{Plant and insect growth conditions.}

A. thaliana ecotype Col-0 and mutants were grown as described previously (Reymond et al. 2000). Mutant seed (ein21, npr 1-1, aba2-1, and aba3-1) were obtained from the Nottingham Arabidopsis Stock Center. Seed of coil-1 (nonglabrous) were a gift from J. Glazebrook (University of Minnesota, St. Paul, MN, U.S.A.) and sid2-1 from C. Nawrath (University of Lausanne, Lausanne, Switzerland). Homozygous coil-1 mutants were selected on plates containing $50 \mu \mathrm{M}$ JA as described previously (Xie et al. 1998) and were transferred to soil after 10 days of growth. S. littoralis (Egyptian cotton worm) eggs were obtained from Syngenta (Stein, Switzerland). Rearing and handling of $P$. rapae (small cabbage white butterfly) and S. littoralis larvae were described previously (Reymond et al. 2004).

\section{Plant treatments.}

Plants were 6 weeks old at the time of treatment. All experiments were biologically independent; they were carried out with several plantings done at intervals of several weeks. For insect challenge, two to three fourth- or fifth-instar larvae were allowed to feed on a plant for 4 to $5 \mathrm{~h}$ in a growth chamber $\left(22^{\circ} \mathrm{C}, 65 \%\right.$ relative humidity, $\left.100 \mu \mathrm{mol} \mathrm{m}{ }^{-2} \mathrm{~s}^{-1}\right)$ until approximately $20 \%$ of leaf area was removed. For each experiment, damaged leaf tissue from 12 challenged plants was harvested and immediately stored in liquid nitrogen. Leaves from 12 
control, unchallenged plants were collected at the same time. Measurements with wild-type plants were done with at least six independent biological replicates; for measurements with all the mutants, at least three independent biological replicates were used. For longer feeding experiments, newly hatched larvae (three for two plants) were allowed to feed continuously during 8 days until the leaves were harvested. For these experiments, there were five replicates for wild-type and two replicates for the aba2-1 mutant. For drought treatment, six-weekold plants were left without water for 5 to 6 days until wilting symptoms appeared (three biological replicates).

\section{Insect bioassays.}

Evaluation of insect performance with $P$. rapae was described previously (Reymond et al. 2004). Briefly, 5-week-old plants were placed in a growth chamber $\left(20^{\circ} \mathrm{C}, 65 \%\right.$ relative humidity, $100 \mu \mathrm{mol} \mathrm{m} \mathrm{m}^{-1} \mathrm{~s}^{-1}$, photoperiod of $10 \mathrm{~h}$ of light and $14 \mathrm{~h}$ of darkness) and one newly hatched larva randomly sampled from the insect colony was placed on each plant (approximately 30 plants per genotype). For bioassays with $S$. littoralis, plants of the same genotype were placed in a Plexiglas box to prevent larvae from moving from one genotype to the other. Thirtythree newly hatched larvae were placed on 22 plants and, after 7 or 8 days of feeding, larvae were removed and weighed to the nearest $0.01 \mathrm{mg}$ using a Mettler-Toledo MT5 balance (Mettler-Toledo, Greifensee, Switzerland).

Caterpillar weights at egg hatch were assumed to be equal and thus only final weights (mean \pm standard error) were measured. For the comparison of several independent biological replicates, relative increase in larval weight (mean \pm standard error) was calculated for each genotype comparison. In the case of experiments comparing two genotypes, data were analyzed using a Student's $t$ test. In the case of experiments comparing more than two genotypes, data were analyzed on log-transformed values by analysis of variance using $\mathrm{R}$ version 1.13 for Mac (Ross and Robert 1996). Where significant $F$ values were found, Tukey's highly significant difference multiple comparison tests were conducted to test for significant differences between individual treatments (Zar 1999). All experiments were repeated several times in independent biological replicates.

\section{Microarray experiments.}

A custom-made microarray was constructed containing 279 cDNAs representing 222 unique genes. EST were obtained from the Arabidopsis Biological Resource Center (Columbus, OH). Insect-regulated genes were identified in a study using a $12 \mathrm{~K}$ Arabidopsis cDNA microarray (Reymond et al. 2004). cDNAs for housekeeping genes and for defense-related genes selected from the literature also were included. All EST were sequenced to confirm gene identity. Preparation of DNA, printing of cDNA microarrays, and labeling of RNA samples was carried out as described previously (Reymond et al. 2004). Microarrays were scanned with a ScanArray 4000 (Packard BioScience SA, Zurich, Switzerland). Photomultiplier and laser power settings were adjusted so that the expression ratio of housekeeping genes was as close to 1.0 as possible and signal intensities were below saturation of the scanner. The average fluorescence intensity for each fluor and for each gene was determined using the ImaGene program (BioDiscovery Inc., Los Angeles). The median background fluorescence signal around each gene spot was subtracted from each spot. To adjust signal intensities between $\mathrm{Cy} 3$ and Cy5 channels, a normalization factor was calculated from the expression ratios of 17 housekeeping genes so that the median expression ratio of these genes was equal to 1.0. This normalization factor then was applied to all background-corrected Cy5 intensities. Signal values $<1,000$ (two to three times the average background intensity) were raised to 1,000 to avoid extreme expression ratios. Because some genes are represented by different EST, the relative change in RNA abundance for one gene could vary between EST, although the trend was always the same. One reason for this variability could be that different EST might correspond to different cDNA lengths, thus changing the hybridization specificities of the probe.

\section{Microarray data analysis.}

Normalized signal intensities were used to calculate expression ratios. To estimate the individual variability of transcript levels between groups of untreated plants, a control hybridization was performed using two independent samples obtained from plants harvested the same day. This experiment was replicated four times on four different days and allowed us to select a twofold change in relative RNA abundance as a robust threshold to identify differentially regulated genes, as previously described (Reymond et al. 2004). In addition, a Student's $t$ test (two sample hypothesis, equal variance) was conducted between $\log _{2}$-transformed expression ratios from control versus control experiments and $\log _{2}$-transformed ratios from control versus treated experiments. Up- and downregulated genes were selected based on a threshold of twofold change in transcript levels and a $P$ value $<0.05$.

To identify genes differentially regulated by herbivory between genotypes, a Student's $t$ test was performed between $\log _{2}$-transformed expression ratios from Col-0 experiments (six to eight replicates) and $\log _{2}$-transformed expression ratios from mutant experiments (three to four replicates). Only genes with an expression ratio $>2$ in at least one genotype and a $P$ value $<0.05$ were considered.

To address the issue of multiple comparisons, an FDR was calculated using the method of Storey and Tibshirani (2003). This method computes a $q$ value for each gene using the distribution of $P$ values of all measurements. For data with $P$. rapae, the FDR at $P=0.05$ was $0.5 \%$ (wild type), $1.5 \%$ (coil-1), $0.9 \%$ (sid2-1), $1.1 \%$ (ein2-1), and $1.2 \%$ (aba2-1). For data with $S$. littoralis, the FDR at $P=0.05$ was $0.7 \%$ (wild type, 5 h), $0.1 \%$ (wild type, 8 days), $2.5 \%$ (coil-1), $1.4 \%$ (sid2-1), $2.3 \%$ (ein2-1), and $0.8 \%$ (aba2-1).

All microarray data have been deposited in ArrayExpress under accession number E-MEXP-1049.

\section{Reverse-transcription PCR analysis.}

For quantitative real-time PCR reactions, RNA samples first were reverse transcribed using Superscript II (Invitrogen, Carlsbad, CA, U.S.A.) based on the manufacturer's instructions with $5 \mu \mathrm{g}$ of total RNA (purified with an RNeasy Qiagen kit followed by a DNaseI treatment) in a final volume of 100 $\mu \mathrm{l}$. The resulting cDNA samples were stored at $-80^{\circ} \mathrm{C}$. Reverse-transcription PCR was performed in triplicate using FullVelocity SYBR green kit (Stratagene, La Jolla, CA, U.S.A.). In a $25-\mu$ reaction volume, $1 \mu \mathrm{l}$ of the cDNA sample was combined with $3.75 \mu \mathrm{l}$ of $\operatorname{ROX}$ (1/5,000 dilution), $12.5 \mu \mathrm{l}$ of $2 \times \mathrm{SYBR}$, and $2.5 \mu \mathrm{l}$ of primer mix where both forward and reverse primers are at a $1-\mu \mathrm{M}$ concentration. The reaction was placed in a MX300 real-time PCR machine (Agilent Technologies, Santa Clara, CA, U.S.A.) with a temperature profile of $95^{\circ} \mathrm{C}$ for $5 \mathrm{~min}$, then 40 cycles of $95^{\circ} \mathrm{C}$ for $10 \mathrm{~s}$ and $55^{\circ} \mathrm{C}$ for $30 \mathrm{~s}$. The following gene specific primers were used: $E I F 4 A 1$, At3g13920-forward (5'-CCAGAAGGCACACAGTTTGATG CA-3'), At3g13920-reverse (5'-AGACTGAGCCTGTTGAAT CACATC-3'); SYP121, At3g11820-forward (5'-GTCTCAGGA AGAAATTGATGGACT-3'), At3g11820-reverse (5'-CCTTTC TTGAATCTCGTTAATGGT-3'); disease resistance protein, At1g72950-forward (5'-TGGATCCGTGTCATGTGAGGAGA C-3') At1g72950-reverse (5'-TGTGCATCAATCCCTTCAAG GTCA-3'); and $\mathrm{TCH} 3$, At2g41100-forward (5'-GATCAAGC 
GCCGCGTCACACTAAA-3'), At2g41100-reverse (5'-TCAGG GAAATCGATGGTTCCATCACC- ${ }^{\prime}$ ). Relative mRNA abundance was calculated using the comparative $\Delta$ cycle threshold method. The values were normalized to the constitutively expressed gene EIF4A1 (At3g13920) and to the control sample (plants without caterpillars).

\section{ACKNOWLEDGMENTS}

This project was supported by a genomics grant from the University of Lausanne. The authors gratefully acknowledge D. Little and C. Darimont for their assistance in rearing insects, B. Kunstner for maintenance of the plants, R. Liechti for establishing the Nomad database, and H.-P. Suter for help with the use of the R statistics software. R. Reist (Syngenta, Stein, Switzerland) kindly provided Spodoptera littoralis eggs. We thank J. Glazebrook for the coil-1 nonglabrous mutant and C. Nawrath for the sid2-1 mutant. We are grateful to D. Little, R. Hammond, and F. Masclaux for helpful comments on the project.

\section{LITERATURE CITED}

Agrawal, A. A., and Kurashige, N. S. 2003. A role for isothiocyanates in plant resistance against the specialist herbivore Pieris rapae. J. Chem. Ecol. 29: 1403-1415.

Anderson, J. P., Badruzsaufari, E., Schenk, P. M., Manners, J. M., Desmond, O. J., Ehlert, C., Maclean, D. J., Ebert, P. R., and Kazan, K. 2004. Antagonistic interaction between abscisic acid and jasmonate-ethylene signaling pathways modulates defense gene expression and disease resistance in Arabidopsis. Plant Cell 16:3460-3479.

Bernays, E. A. 1998. Evolution of feeding behavior in insect herbivores. Bioscience 48:35-44.

Cao, H., Bowling, S. A., Gordon, A. S., and Dong, X. 1994. Characterization of an Arabidopsis mutant that is nonresponsive to inducers of systemic acquired resistance. Plant Cell 6:1583-1592.

Christmann, A., Moes, D., Himmelbach, A., Yang, Y., Tang, Y., and Grill, E. 2006. Integration of abscisic acid signalling into plant responses. Plant Biol. 8:314-325.

Cipollini, D., Enright, S., Traw, M. B., and Bergelson, J. 2004. Salicylic acid inhibits jasmonic acid-induced resistance of Arabidopsis thaliana to Spodoptera exigua. Mol. Ecol. 13:1643-1653.

Collins, N. C., Thordal-Christensen, H., Lipka, V., Bau, S., Kombrink, E., Qiu, J. L., Huckelhoven, R., Stein, M., Freialdenhoven, A., Somerville, S. C., and Schulze-Lefert, P. 2003. SNARE-protein-mediated disease resistance at the plant cell wall. Nature 425:973-977.

Constabel, C. P., Yip, L., Patton, J. J., and Christopher, M. E. 2000. Polyphenol oxidase from hybrid poplar. Cloning and expression in response to wounding and herbivory. Plant Physiol. 124:285-295.

Cooper, W. R., and Goggin, F. 2005. Effects of jasmonate-induced defences in tomato on the potato aphid, Macrosiphum euphorbiae. Entomol. Exp. Appl. 85:121-133.

Cui, J. P., Jander, G., Racki, L. R., Kim, P. D., Pierce, N. E., and Ausubel, F. M. 2002. Signals involved in Arabidopsis resistance to Trichoplusia $n i$ caterpillars induced by virulent and avirulent strains of the phytopathogen Pseudomonas syringae. Plant Physiol. 129:551-564.

Cui, J., Bahrami, A. K., Pringle, E. G., Hernandez-Guzman, G., Bender, C. L., Pierce, N. E., and Ausubel, F. M. 2005. Pseudomonas syringae manipulates systemic plant defenses against pathogens and herbivores. Proc. Natl. Acad. Sci. U.S.A 102:1791-1796.

De Bruxelles, G. L. and Roberts, M. R. 2001. Signals regulating multiple responses to wounding and herbivores. Crit. Rev. Plant Sci. 20:487-521.

De Vos, M., Van Oosten, V. R., Van Poecke, R. M. P., Van Pelt, J. A., Pozo, M. J., Mueller, M. J., Buchala, A. J., Metraux, J. P., Van Loon, L. C., Dicke, M., and Pieterse, C. M. J. 2005. Signal signature and transcriptome changes of Arabidopsis during pathogen and insect attack. Mol. Plant-Microbe Interact. 18:923-937.

De Vos, M., Van Zaanen, W., Koornneef, A., Korzelius, J. P., Dicke, M., Van Loon, L. C., and Pieterse, C. M. 2006. Herbivore-induced resistance against microbial pathogens in Arabidopsis. Plant Physiol. 142:352-363.

Dicke, M., van Poecke, R. M. P., and de Boer, J. G. 2003. Inducible indirect defence of plants: from mechanisms to ecological functions. Basic Appl. Ecol. 4:27-42.

Doares, S. H., Narvaes-Vasquez, J., Conconi, A., and Ryan, C. A. 1995. Salicylic acid inhibits synthesis of proteinase inhibitors in tomato leaves induced by systemin and jasmonic acid. Plant Physiol. 108:1741-1746.

D'Ovidio, R., Raiola, A., Capodicasa, C., Devoto, A., Pontiggia, D., Roberti, S., Galletti, R., Conti, E., O'Sullivan, D., and De Lorenzo, G. 2004. Characterization of the complex locus of bean encoding polyga- lacturonase-inhibiting proteins reveals subfunctionalization for defense against fungi and insects. Plant Physiol. 135:2424-2435.

Ehrlich, P. R., and Raven, P. H. 1964. Butterflies and plants-A study in coevolution. Evolution 18:586-608.

Ellis, C., Karafyllidis, L., and Turner, J. G. 2002. Constitutive activation of jasmonate signaling in an Arabidopsis mutant correlates with enhanced resistance to Erysiphe cichoracearum, Pseudomonas syringae, and Myzus persicae. Mol. Plant-Microbe Interact. 15:1025-1030.

Felton, G. W., and Korth, K. L. 2000. Trade-offs between pathogen and herbivore resistance. Curr. Opin. Plant Biol. 3:309-314.

Fidantsef, A. L., Stout, M. J., Thaler, J. S., Duffey, S. S., and Bostock, R. M. 1999. Signal interactions in pathogen and insect attack: expression of lipoxygenase, proteinase inhibitor II, and pathogenesis-related protein P4 in the tomato, Lycopersicon esculentum. Physiol. Mol. Plant Pathol. 54:97-114.

Gao, L.-L., Anderson, J. P., Klinger, J. P., Nair, R. M., Edwards, O. R., and Singh, K. B. 2007. Involvement of the octadecanoid pathway in bluegreen aphid resistance in Medicago truncatula. Mol. Plant-Microbe Interact. 20:82-93.

Girard, C., and Jouanin, L. 1999. Molecular cloning of cDNAs encoding a range of digestive enzymes from a phytophagous beetle, Phaedon cochleariae. Insect Biochem. Mol. Biol. 29:1129-1142.

Glazebrook, J. 2001. Genes controlling expression of defense responses in Arabidopsis-2001 status. Curr. Opin. Plant Biol. 4:301-308.

Gonzalez-Guzman, M., Apostolova, N., Belles, J. M., Barrero, J. M., Piqueras, P., Ponce, M. R., Micol, J. L., Serrano, R., and Rodriguez, P. L. 2002. The short-chain alcohol dehydrogenase ABA2 catalyzes the conversion of xanthoxin to abscisic aldehyde. Plant Cell 14:1833-1846.

Gupta, V., Willits, M. G., and Glazebrook, J. 2000. Arabidopsis thaliana EDS4 contributes to salicylic acid (SA)-dependent expression of defense responses: evidence for inhibition of jasmonic acid signaling by SA. Mol. Plant-Microbe Interact. 13:503-511.

Guzman, P., and Ecker, J. R. 1990. Exploiting the triple response of Arabidopsis to identify ethylene-related mutants. Plant Cell 2:513-523.

Howe, G. A., Lightner, J., Browse, J., and Ryan, C. A. 1996. An octadecanoid pathway mutant (JL5) of tomato is compromised in signaling for defense against insect attack. Plant Cell 8:2067-2077.

Hui, D., Iqbal, J., Lehmann, K., Gase, K., Saluz, H. P., and Baldwin, I. T. 2003. Molecular interactions between the specialist herbivore Manduca sexta (Lepidoptera, Sphingidae) and its natural host Nicotiana attenuata: V. Microarray analysis and further characterization of large-scale changes in herbivore-induced mRNAs. Plant Physiol. 131:1877-1893.

Johnson, R., Narvaez, J., An, G., and Ryan, C. 1989. Expression of proteinase inhibitors I and II in transgenic tobacco plants: effects on natural defense against Manduca sexta larvae. Proc. Natl. Acad. Sci. U.S.A 86:9871-9875.

Jones, A. M. E., Thomas, V., Truman, B., Lilley, K., Mansfield, J., and Grant, M. 2004. Specific changes in the Arabidopsis proteome in response to bacterial challenge: differentiating basal and R-gene mediated resistance. Phytochemistry 65:1805-1816.

Kahl, J., Siemens, D. H., Aerts, R. J., Gabler, R., Kuhnemann, F., Preston, C. A., and Baldwin, I. T. 2000. Herbivore-induced ethylene suppresses a direct defense but not a putative indirect defense against an adapted herbivore. Planta 210:336-342.

Karban, R., and Baldwin, I. T. 1997. Induced Responses to Herbivory. University of Chicago Press, Chicago.

Katagiri, F. 2004. A global view of defense gene expression regulation-A highly interconnected signaling network. Curr. Opin. Plant Biol. 7:506511.

Kempema, L. A., Cui, X., Holzer, F. M., and Walling, L. L. 2007. Arabidopsis transcriptome changes in response to phloem-feeding silverleaf whitefly nymphs. Similarities and distinctions in response to aphids. Plant Physiol. 143:849-865.

Kessler, A., Halitschke, R., and Baldwin, I. T. 2004. Silencing the jasmonate cascade: induced plant defenses and insect populations. Science 305:665-668

Kliebenstein, D., Pedersen, D., Barker, B., and Mitchell-Olds, T. 2002. Comparative analysis of quantitative trait loci controlling glucosinolates, myrosinase and insect resistance in Arabidopsis thaliana. Genetics 161:325-332.

Kloek, A. P., Verbsky, M. L., Sharma, S. B., Schoelz, J. E., Vogel, J., Klessig, D. F., and Kunkel, B. N. 2001. Resistance to Pseudomonas syringae conferred by an Arabidopsis thaliana coronatine-insensitive (coil) mutation occurs through two distinct mechanisms. Plant J. 26:509-522.

Korth, K. L. 2003. Profiling the response of plants to herbivorous insects. Genome Biol. 4: 221. doi:10.1186/gb-2003-4-7-221. Published online.

Kunkel, B. N., and Brooks, D. M. 2002. Cross talk between signaling pathways in pathogen defense. Curr. Opin. Plant Biol. 5:325-331.

Leon-Kloosterziel, K. M., Gil, M. A., Ruijs, G. J., Jacobsen, S. E., Olszewski, N. E., Schwartz, S. H., Zeevaart, J. A., and Koornneef, M. 
1996. Isolation and characterization of abscisic acid-deficient Arabidopsis mutants at two new loci. Plant J. 10:655-661.

Li, L., Zhao, Y. F., McCaig, B. C., Wingerd, B. A., Wang, J. H., Whalon, M. E., Pichersky, E., and Howe, G. A. 2004. The tomato homolog of CORONATINE-INSENSITIVE1 is required for the maternal control of seed maturation, jasmonate-signaled defense responses, and glandular trichome development. Plant Cell 16:126-143.

Liu, Y. L., Ahn, J. E., Datta, S., Salzman, R. A., Moon, J., HuyghuesDespointes, B., Pittendrigh, B., Murdock, L. L., Koiwa, H., and ZhySalzman, K. 2005. Arabidopsis vegetative storage protein is an antiinsect acid phosphatase. Plant Physiol. 139:1545-1556.

Lorenzo, O., Chico, J. M., Sanchez-Serrano, J. J., and Solano, R. 2004. Jasmonate-insensitive1 encodes a MYC transcription factor essential to discriminate between different jasmonate-regulated defense responses in Arabidopsis. Plant Cell 16:1938-1950.

Mauch-Mani, B., and Mauch, F. 2005. The role of abscisic acid in plantpathogen interactions. Curr. Opin. Plant Biol. 8:409-414.

McCloud, E. S., and Baldwin, I. T. 1997. Herbivory and caterpillar regurgitants amplify the wound-induced increases in jasmonic acid but not nicotine in Nicotiana sylvestris. Planta 203:430-435.

McConn, M., Creelman, R. A., Bell, E., Mullet, J. E., and Browse, J. 1997. Jasmonate is essential for insect defense in Arabidopsis. Proc. Natl. Acad. Sci. U.S.A. 94:5473-5477.

Mewis, I., Appel, H. M., Hom, A., Raina, R., and Schultz, J. C. 2005. Major signaling pathways modulate Arabidopsis glucosinolate accumulation and response to both phloem-feeding and chewing insects. Plant Physiol. 138:1149-1162.

Moran, P. J., and Thompson, G. A. 2001. Molecular responses to aphid feeding in Arabidopsis in relation to plant defense pathways. Plant Physiol. 125:1074-1085.

Moran, P. J., Cheng, Y., Cassell, J. L., and Thompson, G. A. 2002. Gene expression profiling of Arabidopsis thaliana in compatible plant-aphid interactions. Arch. Insect. Biochem. Physiol. 51:182-203.

Musser, R. O., Hum-Musser, S. M., Eichenseer, H., Peiffer, M., Ervin, G. Murphy, J. B., and Felton, G. W. 2002. Herbivory: caterpillar saliva beats plant defences. Nature 416:599-600.

Nawrath, C., and Metraux, J. P. 1999. Salicylic acid induction-deficient mutants of Arabidopsis express PR-2 and PR-5 and accumulate high levels of camalexin after pathogen inoculation. Plant Cell 11:1393-1404.

Pechan, T., Cohen, A., Williams, W. P., and Luthe, D. S. 2002. Insect feeding mobilizes a unique plant defense protease that disrupts the peritrophic matrix of caterpillars. Proc. Natl. Acad. Sci. U.S.A. 99:13319-13323.

Pieterse, C. M. J., and van Loon, L. C. 1999. Salicylic acid-independent plant defence pathways. Trends Plant Sci. 4:52-58.

Ratzka, A., Vogel, H., Kliebenstein, D. J., Mitchell-Olds, T., and Kroymann, J. 2002. Disarming the mustard oil bomb. Proc. Natl. Acad. Sci. U.S.A. 99:11223-11228.

Reymond, P., and Farmer, E. E. 1998. Jasmonate and salicylate as global signals for defense gene expression. Curr. Opin. Plant Biol. 1:404-411.

Reymond, P., Weber, H., Damond, M., and Farmer, E. E. 2000. Differential gene expression in response to mechanical wounding and insect feeding in Arabidopsis. Plant Cell 12:707-720.

Reymond, P., Bodenhausen, N., Van Poecke, R. M., Krishnamurthy, V., Dicke, M., and Farmer, E. E. 2004. A conserved transcript pattern in response to a specialist and a generalist herbivore. Plant Cell 16:3132-3147.

Ross, I., and Robert, G. 1996. R: A language for data analysis and graphics. J Comput. Graph. Stat. 5:299-314.

Royo, J., Leon, J., Vancanneyt, G., Albar, J. P., Rosahl, S., Ortego, F., Castanera, P., and Sanchez-Serrano, J. J. 1999. Antisense-mediated depletion of a potato lipoxygenase reduces wound induction of proteinase inhibitors and increases weight gain of insect pests. Proc. Natl. Acad. Sci. U.S.A. 96:1146-1151.

Ryan, C. A. 1990. Protease inhibitors in plants: genes for improving defenses against insects and pathogens. Annu. Rev. Phytopathol. 28:245-249.

Schenk, P. M., Kazan, K., Wilson, I., Anderson, J. P., Richmond, T., Somerville, S. C., and Manners, J. M. 2000. Coordinated plant defense responses in Arabidopsis revealed by microarray analysis. Proc. Natl. Acad. Sci. U.S.A. 97:11655-11660.

Schmidt, D. D., Voelckel, C., Hartl, M., Schmidt, S., and Baldwin, I. T. 2005. Specificity in ecological interactions. Attack from the same lepidopteran herbivore results in species-specific transcriptional responses in two solanaceous host plants. Plant Physiol. 138:1763-1773.

Schoonhoven, L. M., Jermy, T., and Van Loon, J. J. A. 1998. Insect-Plant Biology. From Physiology to Evolution, Chapman and Hall, London.

Shah, J. 2003. The salicylic acid loop in plant defense. Curr. Opin. Plant Biol. 6:365-371.

Shen, Z. C., Reese, J. C., and Reeck, G. R. 1996. Purification and characterization of polygalacturonase from the rice weevil, Sitophilus oryzae (Coleoptera: Curculionidae). Insect Biochem. Mol. Biol. 26:427-433.
Stintzi, A., Weber, H., Reymond, P., Browse, J., and Farmer, E. E. 2001. Plant defense in the absence of jasmonic acid: the role of cyclopentenones. Proc. Natl. Acad. Sci. U.S.A. 98:12837-12842.

Storey, J. D., and Tibshirani, R. 2003. Statistical significance for genomewide studies. Genome Biol. 100:9440-9445.

Stotz, H. U., Pittendrigh, B. R., Kroymann, J., Weniger, K., Fritsche, J. Bauke, A. and Mitchell-Olds, T. 2000. Induced plant defense responses against chewing insects. Ethylene signaling reduces resistance of Arabidopsis against Egyptian cotton worm but not diamondback moth. Plant Physiol. 124:1007-1018.

Stotz, H. U., Koch, T., Biedermann, A., Weniger, K., Boland, W., and Mitchell-Olds, T. 2002. Evidence for regulation of resistance in Arabidopsis to Egyptian cotton worm by salicylic and jasmonic acid signaling pathways. Planta 214:648-652.

Thaler, J. S., and Bostock R. M. 2004. Interactions between abscisic-acidmediated responses and plant resistance to pathogenes and insects. Ecology 85:48-58.

Thaler, J. S., Farag, M. A., Pare, P. W., and Dicke, M. 2002. Jasmonate-deficient plants have reduced direct and indirect defences against herbivores. Ecol. Lett. 5:764-774.

Thompson, G. A., and Goggin, F. L. 2006. Transcriptomics and functional genomics of plant defence induction by phloem-feeding insects. J. Exp. Bot. 57:755-766.

Ton, J., Jakab, G., Toquin, V., Flors, V., Iavicoli, A., Maeder, M. N., Metraux, J. P., and Mauch-Mani, B. 2005. Dissecting the $\beta$-aminobutyric acid-induced priming phenomenon in Arabidopsis. Plant Cell 17:987-999.

van de Ven, W. T. G. , LeVesque, C. S., Perring, T. M., and Walling, L. L. 2000. Local and systemic changes in squash gene expression in response to silverleaf whitefly feeding. Plant Cell 12:1409-1423.

Van Poecke, R. M. P., and Dicke, M. 2002. Induced parasitoid attraction by Arabidopsis thaliana: involvement of the octadecanoid and the salicylic acid pathway. J. Exp. Bot. 53:1793-1799.

Varet, A., Parker, J., Tornero, P., Nass, N., Nurnberger, T., Dangl, J. L., Scheel, D., and Lee, J. 2002. NHL25 and NHL3, two NDR1/HIN1-like genes in Arabidopsis thaliana with potential role(s) in plant defense. Mol. Plant-Microbe Interact. 15:608-616.

Voelckel, C., and Baldwin, I. T. 2004. Herbivore-induced plant vaccination. Part II. Array-studies reveal the transience of herbivore-specific transcriptional imprints and a distinct imprint from stress combinations. Plant J. 38:650-663.

Voelckel, C., Weisser, W. W., and Baldwin, I. T. 2004. An analysis of plant-aphid interactions by different microarray hybridization strategies. Mol. Ecol. 13:3187-3195.

Walling, L. L. 2000. The myriad plant responses to herbivores. J. Plant Growth Regul. 19:195-216.

Wittstock, U., Agerbirk, N., Stauber, E. J., Olsen, C. E., Hippler, M., Mitchell-Olds, T., Gershenzon, J., and Vogel, H. 2004. Successful herbivore attack due to metabolic diversion of a plant chemical defense. Proc. Natl. Acad. Sci. U.S.A. 101:4859-4864.

Xie, D. X., Feys, B. F., James, S., Nieto-Rostro, M., and Turner, J. G. 1998. COI1: an Arabidopsis gene required for jasmonate-regulated defense and fertility. Science 280:1091-1094.

Xiong, L. M., Ishitani, M., Lee, H., and Zhu, J. K. 2001. The Arabidopsis LOS5/ABA3 locus encodes a molybdenum cofactor sulfurase and modulates cold stress- and osmotic stress-responsive gene expression. Plant Cell 13:2063-2083.

Zar, J. H. 1999. Biostatistical Analysis, 4th ed. Prentice Hall, Englewood Cliffs, NJ, U.S.A.

Zarate, S. I., Kempema, L. A., and Walling, L. L. 2007. Silverleaf whitefly induces salicylic acid defenses and suppresses effectual jasmonic acid defenses. Plant Physiol. 143:866-875.

Zhang, B., Ramonell, K., Somerville, S., and Stacey, G. 2002. Characterization of early, chitin-induced gene expression in Arabidopsis. Mol. Plant-Microbe Interact. 15:963-970.

Zhao, Y., Thilmony, R., Bender, C. L., Schaller, A., He, S. Y., and Howe, G. A. 2003. Virulence systems of Pseudomonas syringae pv. tomato promote bacterial speck disease in tomato by targeting the jasmonate signaling pathway. Plant J. 36:485-499.

Zhu-Salzman, K., Salzman, R. A., Ahn, J.-E., and Koiwa, H. 2004. Transcriptional regulation of sorghum defense determinants against a phloem-feeding aphid. Plant Physiol. 134:420-431.

Zhu-Salzman, K., Bi, J.-L., and Liu, T.-X. 2005. Molecular strategies of plant defense and insect counter-defense. Insect Sci. 12:3-15.

\section{AUTHOR-RECOMMENDED INTERNET RESOURCE}

ArrayExpress database: www.ebi.ac.uk/arrayexpress 